\title{
DUPIN HYPERSURFACES AND A LIE INVARIANT
}

\author{
By REIKo MiYAOKA
}

Dedicated to Professor Tominosuke Otsuki on his 70th birthday

\section{$\S 1$. Introduction.}

In [9], Pinkall developes a Lie's sphere geometry on hypersurfaces in a space form and successfully applies the theory to a class of hypersurfaces called "Dupin". A Dupin hypersurface is a hypersurface each of which principal curvatures has a constant multiplicity with a vanishing derivative in the corresponding curvature direction. One of his results is the local Lie equivalence of cyclides of Dupin with isoparametric hypersurfaces, where a cyclide of Dupin is a Dupin hypersurface with exactly two principal curvatures. This is essentially used in [4] to find a solution to a simple progressing wave equation.

For any integer $g$, we can construct a Dupin hypersurface with $g$ principal curvatures of arbitrary multiplicities. Isoparametric hypersurfaces, however, have $g \in\{1,2,3,4,6\}$ principal curvatures with non-arbitrary multiplicities if $g \geqq 3$. Thus the equivalence problem between Dupin hypersurfaces and isoparametric hypersurfaces for $g \geqq 3$ requires some more conditions on Dupin hypersurfaces.

In [11], Thorbergsson guarantees coincidence of compact embedded Dupin hypersurfaces with isoparametric hypersurfaces in cohomology level. This motivates Cecil and Ryan a conjecture [3]: A compact embedded Dupin hypersurface is Lie equivalent to an isoparametric hypersurface. Besides the trivial case $g=1$, this is already known true when $g=2[2]$. For $g=3$, the author gives a positive answer in [4]. In this paper, we find a certain Lie invariant by which we get a non-trivial necessary condition for the equivalence when $g=4$ and 6. A sufficient condition for $g=4$ is obtained as well, and in the forthcoming paper, we give it for $g=6$.

After this paper was finished, Pinkall and Thorbergsson construct counterexamples to the conjecture for $g=4[10]$. Independently, Ozawa and the author get counterexamples for $g=4$ and 6 in [6], using a new method producing taut embeddings. Both examples are shown to be not Lie equivalent to isoparametric hypersurfaces by using the Lie invariant obtained in the present paper.

Received June 8, 1988; revised November 21, 1988 
Now our results are:

THEOREM I. Let $\lambda, \mu, \nu, \tau$ be distinct principal curvatures of a hypersurface $M$ in a space form $\bar{M}(c)$. Then the function

$$
\Psi=\frac{(\lambda-\mu)(\tau-\nu)}{(\lambda-\nu)(\tau-\mu)}
$$

is invariant under Lie transformations.

An immediate consequence of this theorem is:

CoRollaly. For a Dupin hypersurface $M$ in $\bar{M}(c)$ with four or six principal curvatures to be Lie equivalent to an isoparametric hypersurface, the function $\Psi$ must be constant on $M$ for any distinct four principal curvatures $\lambda$, $\mu, \nu, \tau$ of $M$.

As for a sufficient condition, we have:

THEOREM II. Let $M$ be a compact embedded Dupin hyperface with four principal curvatures $\lambda>\mu>\nu>\tau$ in $\bar{M}(c)$. If $\Psi$ is constant on $M$ and if for each $\lambda$-leaf $L^{\lambda}\left(\tau\right.$-leaf $L^{\tau}$, resp.), there exists a $\nu$-leaf $L_{\lambda}^{\nu}\left(\mu\right.$-leaf $L_{\tau}^{\mu}$, resp. $)$ such that $L_{q}^{\mu} \cap L_{\lambda}^{\nu} \neq \varnothing$ for all $q \in L^{\lambda}\left(L_{q}^{\nu} \cap L_{\tau}^{\mu} \neq \varnothing\right.$ for all $q \in L^{\tau}$, resp. $)$, then $M$ is Lie equivalent to an isoparametric hypersurface in a sphere.

In $\S 2-3$, we give a summery of Lie's sphere geometry, and prove Theorem I in $\S 4$. In $\S 5-7$, we investigate compact embedded Dupin hypersurfaces with four principal curvatures. We use the constantness of $\Psi$ in $\S 8-9$. The assump tion on leaves is used in the last section to prove Theorem II.

\section{§ 2. Definitions.}

Let $\bar{M}(c)$ be an $n$-dimensional complete simply-connected space form of constant curvature $c$. An immersed hypersurface $M$ in $\bar{M}(c)$ is called Dupin if it satisfies :

(i) The multiplicity of each principal curvature is constant on $M$.

(ii) Each principal curvature is constant along its leaf of the corresponding curvature distribution.

By an isoparametic hypersurface, we mean an immersed hypersurface in $\bar{M}(c)$ with constant principal curvatures. Obviously, isoparametric hypersurfaces are Dupin, but a (non-isometric) conformal image of an isoparametric hypersurface and its parallel hypersurfaces are non-isoparametric Dupin hypersurfaces.

By conformal invariance of Dupin hypersurfaces and the function $\Psi$, we discuss on the objects in $S^{n}=\bar{M}(1)$ in this paper.

$A$ Lie transformation is a transformation on a space of all oriented hyper- 
shperes in $S^{n}$ that preserves oriented contact (see $\S 3$ ).

An immersed submanifold $X$ in $S^{n}$ is said to be taut, if for all $x \in S^{n}$ such that $d_{x}:=d(x,)^{2}$ (square of the spherical distance) is a Morse function on $M$, the number of critical points of $d_{x}$ is equal to $\beta\left(M ; Z_{2}\right)$, the sum of $\boldsymbol{Z}_{2}$-Betti numbers of $M$.

Lie invariance of tautness is first mentioned by Pinkall [8], and is proved by Cecil and Chern [1]. Now the important is:

THEOREM (Thorbergsson [11]). A complete embedded Dupin hypersurface $M$ in $\bar{M}(c)$ is taut. If $M$ is compact, then $g$ is $1,2,3,4,6$. Moreover, $H^{*}(M$; $\boldsymbol{Z}_{2}$ ) is obtained by Münzner's method.

This theorem motivates Cecil and Ryan the conjecture, and is essentially used in this paper.

\section{§ 3. Basic facts.}

In this section, we review Lie geometric description of hypersurfaces in $S^{n}$, and give basic facts on Lie transformations. For details, see [9].

Let $S^{n}$ be the unit shpere in $\boldsymbol{R}^{n+1}$. For $k^{t}(z, x, y) \in V_{n+3}:=\boldsymbol{R}^{n+1} \times \boldsymbol{R} \times \boldsymbol{R}$, define the symmetric bilinear form $\langle$,$\rangle on V_{n+3}$ by

$$
\langle k, k\rangle=\|z\|^{2}-x^{2}-y^{2} .
$$

Let $P^{n+2}$ be the real projective space corresponding to $V_{n+3}$ and denote by $[k]$ the projective point spanned by $k \in V_{n+3}$. Then the quadric

$$
Q=\left\{[k] \in P^{n+2} \mid\langle k, k\rangle=0\right\}
$$

is identified with the set of all oriented hyperspheres in $S^{n}$. Two oriented hyperspheres $\left[k_{1}\right]$ and $\left[k_{2}\right]$ are in oriented contact iff $\left\langle k_{1}, k_{2}\right\rangle=0$, which is equivalent to that the projective line in $P^{n+2}$ spanned by [ $\left.k_{1}\right]$ and $\left[k_{2}\right]$ is entirely contained in $Q$. A Lie transformation of $S^{n}$ is interpreted as a line preserving diffeo $\varphi: Q \rightarrow Q$. This is known to be the restriction to $Q$ of an projective transformation $\alpha: P^{n+2} \rightarrow P^{n+2}$ leaving $Q$ fixed [9, Lemma 4]. Therefore, a Lie transformation is represented by $A=\left(a_{1}, a_{2}, \cdots, a_{n+3}\right) \in O(n+1,2)$, where $\left\{a_{1}, a_{2}, \cdots, a_{n+3}\right\}$ is the so-called Lie frame, i. e. an ordered set of vectors in $V_{n+3}$ satisfying

$$
\left(\left\langle a_{\imath}, a_{\jmath}\right\rangle\right)_{1 \leq \imath, \jmath \leq n+3}=\left(\begin{array}{rrr}
I_{n+1} & \multicolumn{2}{c}{0} \\
& -1 & 0 \\
0 & 0 & -1
\end{array}\right) .
$$

LEMma 3.1. $A$ is in $O(n+1,2)$ iff ${ }^{t} A \in O(n+1,2)$. In particular, both ordered sets of colume vectors and line vectors of $A$ are Lie frames of $V_{n+3}$.

Proof. Put 


$$
A=\left(\begin{array}{cc}
A_{1} & A_{2} \\
A_{3} & A_{4}
\end{array}\right)
$$

where $A_{1} \in M_{n+1}(\boldsymbol{R}), A_{2},{ }^{t} A_{3} \in M_{n+1,2}^{\prime}(\boldsymbol{R}), A_{4} \in M_{2}(\boldsymbol{R})$. Define

$$
\tilde{A}=\left(\begin{array}{cc}
A_{1} & \sqrt{-1} A_{2} \\
\sqrt{-1} A_{3} & -A_{4}
\end{array}\right) .
$$

Then it is easy to see that $A \in O(n+1,2)$ iff ${ }^{t} \tilde{A} \tilde{A}=I$. Since ${ }^{t} \tilde{A} \tilde{A}=I$ iff $\tilde{A}^{t} \tilde{A}=$ $I$, we get ${ }^{t} A \in O(n+1,2)$ iff $A=O(n+1,2)$.

q.e.d.

Remark 3.2. We obtain Lie frames of $V_{n+3}$ as follows: Choose two linearly independent timelike vectors in $V_{n+3}$ so that they span a timelike plane $T$. Let $S$ be the $(n+1)$-dimensional spacelike subspace orthogonal to $T$ with respect to $\langle$,$\rangle . Since \langle$,$\rangle is definite on S$ and $T$, we can choose $a_{1}, a_{2}, \cdots, a_{n+1} \in S$ and $a_{n+2}, a_{n+3} \in T$ so that

$$
\left(\left\langle a_{\imath}, a_{\jmath}\right\rangle\right)_{1 \leqq \imath, \jmath \leqq n+3}=\left(\begin{array}{rrr}
I_{n+1} & 0 & 0 \\
& -1 & 0 \\
0 & 0 & -1
\end{array}\right),
$$

i.e. $\left\{a_{1}, a_{2}, \cdots, a_{n+3}\right\}$ is a Lie frame of $V_{n+3}$. In particular, det $A \neq 0$ since $a_{1}, \cdots, a_{n+3}$ are linearly independent.

Now, for an $(n-1)$-dimensional manifold $M$ and a pair of mappings $k_{1}, k_{2}$ : $M \rightarrow V_{n+3},\left(M, k_{1}, k_{2}\right)$ is called a Lie geometric hypersurface if it satisfies:

a) For all $p \in M$, the vectors $k_{1}(p)$ and $k_{2}(p)$ are linearly independent and we have

$$
\left\langle k_{\imath}, k_{\jmath}\right\rangle=0, \quad i, j=1,2 .
$$

b) There is no $p \in M, X \in T_{p} M$ such that simultaneously $d k_{1}(X)$ and $d k_{2}(X)$ are in $\operatorname{span}\left(k_{1}(p), k_{2}(p)\right)$.

c) $\left\langle d k_{1}, k_{2}\right\rangle=0$.

Remark 3.3. Obviously, if $\left(M, k_{1}, k_{2}\right)$ is a Lie geometric hypersurface in $S^{n}$, so is $\left(M, A k_{1}, A k_{2}\right)$ for any Lie transformation $A$.

Remark 3.4. The properties a), b), c) are preserved by

$$
\tilde{k}_{1}=\alpha k_{1}+\beta k_{2}, \quad \tilde{k}_{2}=\gamma k_{1}+\delta k_{2}
$$

where $\alpha, \beta, \gamma, \delta: M \rightarrow \boldsymbol{R}$ are functions such that $\alpha \delta-\beta \gamma$ is everywhere different from zero on $M$.

Example 3.5. Let $\boldsymbol{p}: M \rightarrow S^{n}$ be an oriented hypersurface and let $\boldsymbol{n}: M \rightarrow S^{n}$ be defined by $\boldsymbol{n}(p)=$ the unit normal vector at $p \in M$. Then for 


$$
k_{1}={ }^{t}(\boldsymbol{p}, 1,0), \quad k_{2}={ }^{t}(\boldsymbol{n}, 0,1)
$$

$\left(M, k_{1}, k_{2}\right)$ is a Lie geometric hypersurface of $S^{n}$.

LEMMA 3.6. If $\left(M, k_{1}, k_{2}\right)$ is a Lie geometric hypersurface of $S^{n}$, then there are functions $\alpha, \beta, \gamma, \delta: M \rightarrow \boldsymbol{R}$ and maps $\boldsymbol{p}, \boldsymbol{n}: M \rightarrow S^{n}$ such that

$$
\alpha k_{1}+\beta k_{2}={ }^{t}(\boldsymbol{p}, 1,0), \quad \gamma k_{1}+\delta k_{2}={ }^{t}(\boldsymbol{n}, 0,1) .
$$

Proof. Let $k_{1}={ }^{t}\left(\boldsymbol{h}_{1}, a, b\right)$ and $k_{2}={ }^{t}\left(\boldsymbol{h}_{2}, c, d\right)$. Then we have

$$
\begin{aligned}
(a d-b c)^{2} & =\left(a^{2}+b^{2}\right)\left(c^{2}+d^{2}\right)-(a c+b d)^{2} \\
& =\left\|\boldsymbol{h}_{1}\right\|^{2}\left\|\boldsymbol{h}_{2}\right\|^{2}-\left\langle\boldsymbol{h}_{1}, \boldsymbol{h}_{2}\right\rangle^{2} \\
& >0
\end{aligned}
$$

by a). Thus there exists the inverse matrix of $\left(\begin{array}{ll}a & b \\ c & d\end{array}\right)$ which we denote by $\left(\begin{array}{ll}\alpha & \beta \\ \gamma & \delta\end{array}\right)$

q.e.d.

For $\boldsymbol{p}, \boldsymbol{n}: M \rightarrow S^{n}$ in Example 3.5, a parallel hypersurface is defined by

$$
\boldsymbol{p}_{\alpha}=\cos \alpha \boldsymbol{p}+\sin \alpha \boldsymbol{n}, \quad \alpha \in[\pi, \pi) .
$$

of which the unit normal vector field $\boldsymbol{n}_{\alpha}$ is given by

$$
\boldsymbol{n}_{\alpha}=-\sin \alpha \boldsymbol{p}+\cos \alpha \boldsymbol{n} \text {. }
$$

Remark 3.7. $\boldsymbol{p}: M \rightarrow S^{n}$ in Lemma 3.6 is not always an immersion. But singularlities can be removed by passing to a parallel hypersurface of $\boldsymbol{p}[9$, Theorem 1].

Remark 3.8. Define $A \in O(n+1,2)$ by

$$
A=\left(\begin{array}{rrr}
I_{n+1} & 0 & \\
& \cos \alpha & \sin \alpha \\
0 & -\sin \alpha & \cos \alpha
\end{array}\right) .
$$

Then we have for $k_{1}, k_{2}$ in (3.3),

$$
A k_{1}={ }^{t}(\boldsymbol{p}, \cos \alpha,-\sin \alpha), \quad A k_{2}={ }^{t}(\boldsymbol{n}, \sin \alpha, \cos \alpha) .
$$

Using Lemma 3.6, we obtain

$$
\begin{aligned}
& \tilde{k}_{1}=\cos \alpha A k_{1}+\sin \alpha A k_{2}={ }^{t}\left(\boldsymbol{p}_{\alpha}, 1,0\right) \\
& \tilde{k}_{2}=-\sin \alpha A k_{1}+\cos \alpha A k_{2}={ }^{t}\left(\boldsymbol{n}_{\alpha}, 0,1\right) .
\end{aligned}
$$

Thus parallel hypersurfaces are obtained by a Lie transformation of type (3.5). 
Definition. Let $\left(M, k_{1}, k_{2}\right)$ be a Lie geometric hypersurface, $p \in M, \lambda, \mu \in$ $\boldsymbol{R},(\lambda, \mu) \neq(0,0)$. Then the hypersphere $k=\lambda k_{1}(p)+\mu k_{2}(p)$ is called a curvature sphere at $p$ if there is a tangent vector $X \in T_{p} M, X \neq 0$ such that

$$
\lambda d k_{1}(X)+\mu d k_{2}(X) \in \operatorname{span}\left(k_{1}(p), k_{2}(p)\right) .
$$

$X$ is called a direction of curvature corresponding to $k$.

Remark 3.9. The notion of curvature sphere is invariant under Lie transformation. Especially, the direction of a curvature is preserved by Lie transformations. When $\left(M, k_{1}, k_{2}\right)$ is given by (3.3), $k$ is a curvature sphere iff $\tilde{\lambda}=\lambda / \mu$ is a principal curvature of the hypersurface at $p$. The direction of curvature is a principal vector with respect to $\tilde{\lambda}$.

LEMMA 3.10. Let $\left(M, k_{1}, k_{2}\right)$ be a Lie geometric hypersurface of $S^{n}$ aud let $\left(M, \tilde{k}_{1}, \tilde{k}_{2}\right)$ be its another description by (3.2). Then $\lambda k_{1}(p)+\mu k_{2}(p)$ is a curvature sphere at $p \in M$ iff $\sigma \tilde{k}_{1}(p)+\tau \tilde{k}_{2}(p)$ is a curvature sphere at $p$, where

$$
\left(\begin{array}{cc}
\alpha & \gamma \\
\beta & \delta
\end{array}\right)\left(\begin{array}{l}
\sigma \\
\tau
\end{array}\right)=r\left(\begin{array}{l}
\lambda \\
\mu
\end{array}\right), \quad r \in \boldsymbol{R}, r \neq 0 .
$$

Proof. Put $L=\operatorname{span}\left(k_{1}(p), k_{2}(p)\right)=\operatorname{span}\left(\tilde{k}_{1}(p), \tilde{k}_{2}(p)\right)$. Then

$$
\begin{aligned}
\sigma d \tilde{k}_{1}+\tau d \tilde{k}_{2} & \equiv \sigma\left(\alpha d k_{1}+\beta d k_{2}\right)+\tau\left(\gamma d k_{1}+\delta d k_{2}\right)(\bmod L) \\
& =(\alpha \sigma+\gamma \tau) d k_{1}+(\beta \sigma+\delta \tau) d k_{2} .
\end{aligned}
$$$$
\text { q. e.d. }
$$

\section{§4. Principal curvatures of a Lie image.}

In this section, we compute principal curvatures of a Lie image of a hypersurface $M$ in $S^{n}$. To define "a Lie image" precisely, we review the Legendre map from $M$ to the space of lines in $Q$ which we denote by $\Lambda^{2 n-1}$. Then we study about spherical projections from $\Lambda^{2 n-1}$ to a sphere, and show that it sufficient to take a special spherical projection when we study Lie images of $M$.

Definition 4.1. For a hypersurface $M$ in $S^{n}$, let $\left(M, k_{1}, k_{2}\right)$ be the corresponding Lie geometric hypersurface given in (3.3). Then the Legendre map $\mathcal{L}: M \rightarrow \Lambda^{2 n-1}$ is defined for $p \in M$ by $\mathcal{L}(p)=$ the line in $Q$ spanned by $k_{1}(p)$ and $k_{2}(p)$.

Definition 4.2. For an ordered pair $(u, v)$ of unit timelike vectors satisfying $\langle u, v\rangle=0$, the spherical projection

$$
\pi_{u, v}: \Lambda^{2 n-1} \longrightarrow S_{u, v}^{n}
$$

is defined as follows: For $l \in \Lambda^{2 n-1}$, let

$$
l_{1}=l \cap v^{\perp} \quad(\in Q)
$$


which can be defined since $\operatorname{dim}\{\operatorname{span} l\}=2$ and $\operatorname{dim} v^{\perp}=n+2$ in $V_{n+3}$. Then represent $l_{1}$ by the (unique) vector $\tilde{l}_{1}$ in $V_{n+3}$ satisfying $\left\langle\tilde{l}_{1}, u\right\rangle=-1$, which is possible since $l_{1} \in Q$ and $\left\langle l_{1}, v\right\rangle=0$. Then $\pi_{u, v}(l)$ is defined to be the vector $\tilde{l}_{1}-u$. Obviously, $\left\langle\pi_{u, v}(l), \pi_{u, v}(l)\right\rangle=1$, and so it lies in the unit sphere $S_{u, v}^{n}$ of $u^{\perp} \cap v^{\perp}$ in $V_{n+3}$.

Now let $u_{0}=(0,1,0)$ and $v_{0}=(0,0,1)$ in our standard coordinate system of $V_{n+3}$. Note that $S^{n}=S_{u_{0}, v_{0}}^{n}$, and write $\pi_{0}=\pi_{u_{0}, v_{0}}$. For the pair $(u, v)$ above, there is a transformation $A_{0} \in O(n+1,2)$ such that $A_{0} u=u_{0}$ and $A_{0} v=v_{0}$. Let $\tilde{A}_{0}$ be the Lie transformation represented by $A_{0}$. Since $\tilde{A}_{0}$ induces a transformation on $A^{2 n-1}$, it induces a map $f_{A_{0}}: S_{u, v}^{n} \rightarrow S^{n}$, so that the following diagram is commutative:

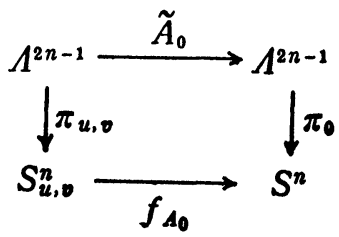

In fact, for $l \in \Lambda^{2 n-1}$ with $\pi_{u, v}(l)=\tilde{l}_{1}-u$, we have

and

$$
0=\left\langle\tilde{l}_{1}, v\right\rangle=\left\langle A_{0} \tilde{l}_{1}, A_{0} v\right\rangle=\left\langle A_{0} \tilde{l}_{1}, v_{0}\right\rangle
$$

$$
-1=\left\langle\tilde{l}_{1}, u\right\rangle=\left\langle A_{0} \tilde{l}_{1}, A_{0} u\right\rangle=\left\langle A_{0} \tilde{l}_{1}, u_{0}\right\rangle
$$

where $\tilde{l}_{1} \in l$, i. e. $\left\langle\tilde{l}_{1}, l\right\rangle=0=\left\langle A_{0} \tilde{l}_{1}, \tilde{A}_{0} l\right\rangle$. So we get

$$
\pi_{0}\left(\tilde{A}_{0} l\right)=A_{0} \tilde{l}_{1}-u_{0}=A_{0}\left(\tilde{l}_{1}-u\right)=A_{0}\left(\pi_{u, v}(l)\right) .
$$

This means that $\pi_{u, v}(l)=\pi_{u, v}\left(l^{\prime}\right)$ leads to $\pi_{0}\left(\tilde{A}_{0} l\right)=\pi_{0}\left(\tilde{A}_{0} l^{\prime}\right)$, that is, $f_{A_{0}}$ is welldefined and $f_{A_{0}}(p)=A_{0} p$ for $p \in S_{u, v}^{n}$. From this follows immediately:

Proposition 4.3. $f_{A_{0}}: S_{u, v}^{n} \rightarrow S^{n}$ is an isometry.

Definition 4.4. For a hypersurface $M$ in $S^{n}$, a Lie image of $M$ is a spherically projected image of $\tilde{A} \mathcal{L}$ where $\mathcal{L}$ is the Legendre map of $M$ and $\tilde{A}$ is some Lie transformation.

From Proposition 4.3, we get:

COROLlaRY 4.5. Lie images of $M$ are obtained, up to isometry, by $\pi_{0}(\tilde{A} \mathcal{L})$ for some $A \in O(n+1,2)$.

Now, we compute principal curvatures of $\pi_{0}(\tilde{A} \mathcal{L})$ where $\tilde{A}$ is the Lie transformation represented by $A \in O(n+1,2)$. For $\left(M, k_{1}, k_{2}\right)$ given by (3.3), we may denote

$$
A k_{1}={ }^{t}\left(\boldsymbol{h}_{1}, a, b\right), \quad A k_{2}={ }^{t}\left(\boldsymbol{h}_{2}, c, d\right)
$$


where $\boldsymbol{h}_{1}, \boldsymbol{h}_{2}: M \rightarrow V_{n+3}$ and $a, b, c, d: M \rightarrow \boldsymbol{R}$. Then we have

$$
\tilde{k}_{1}=\alpha A k_{1}+\beta A k_{2}={ }^{t}(\boldsymbol{q}, 1,0), \quad \tilde{k}_{2}=\gamma A k_{1}+\delta A k_{2}={ }^{t}(\boldsymbol{m}, 0,1)
$$

where $\boldsymbol{q}, \boldsymbol{m}: M \rightarrow S^{n}$ and

$$
\left(\begin{array}{ll}
\alpha & \beta \\
\gamma & \delta
\end{array}\right)=\left(\begin{array}{ll}
a & b \\
c & d
\end{array}\right)^{-1}
$$

in fact, $\pi_{0}(\tilde{A} \mathcal{L})$ is nothing but $\boldsymbol{q}$.

Now let $\lambda$ be a principal curvature of the immersion $\boldsymbol{p}$ at $p \in M$, and assume that $q: M \rightarrow S^{n}$ is an immersion around $p$. Then $\lambda k_{1}(p)+k_{2}(p)$ is the curvature sphere and so is $\lambda A k_{1}(p)+A k_{2}(p)$ (Remark 3.9). Thus by Lemma 3.10, the corresponding principal curvature $\tilde{\lambda}$ of the immersion $\boldsymbol{q}: M \rightarrow S^{n}$ at $\boldsymbol{q}(p)$ is given by $\tilde{\lambda}=\sigma / \tau$, where

$$
\left(\begin{array}{l}
\sigma \\
\tau
\end{array}\right)=r\left(\begin{array}{ll}
\alpha & \gamma \\
\beta & \delta
\end{array}\right)^{-1}\left(\begin{array}{l}
\lambda \\
1
\end{array}\right)=r\left(\begin{array}{ll}
a & c \\
b & d
\end{array}\right)\left(\begin{array}{l}
\lambda \\
1
\end{array}\right)=r\left(\begin{array}{l}
a \lambda+c \\
b \lambda+d
\end{array}\right), \quad r \in \boldsymbol{R}, r \neq 0 .
$$

This implies

$$
\tilde{\lambda}=\frac{a \lambda+c}{b \lambda+d}
$$

Therefore, we get:

Proposition 4.6. Between any principal curvature $\lambda$ of a hypersurface $M$ in $S^{n}$ and the corresponding principal curvature $\tilde{\lambda}$ of a Lie image of $M$, there is a relation (4.2) where $a, b, c, d$ are functions on $M$ determined by the Lie transformation.

Thus we have:

THEOREM I. Let $\lambda, \mu, \nu, \tau$ be distinct principal curvatures of a hypersurface $M$ in $\bar{M}(c)$. Then the function

$$
\Psi=\frac{(\lambda-\mu)(\tau-\nu)}{(\lambda-\nu)(\tau-\mu)}
$$

is invariant under Lie transformations.

Remark 4.7. Note that $\frac{(\lambda-\mu)}{(\lambda-\nu)}$ and $\frac{(\tau-\nu)}{(\tau-\mu)}$ are invariant under conformal transformation and so the result holds for $M$ in $\bar{M}(c)$.

Remark 4.8. It is easily seen that $\Psi$ is the cross ratio among four projective points $k^{\lambda}, k^{\mu}, k^{\nu}, k^{\tau}$ on the line in $Q$ determined by $k_{1}$ and $k_{2}$ given by (3.3), where $k^{*}$ is the curvature sphere with respect to $*$. This is another proof of Theorem I. In the following, we denote the cross ratio $\Psi$ by $[\lambda, \tau ; \mu, \nu]$. 
COROLLARY. For a Dupin hypersurface in a sphere with four or six principal curvatures to be Lie equivalent to an isoparametric hypersurface, the function $\Psi$ must be constant on $M$ for any distinct four principal curvatures $\lambda, \mu, \nu, \tau$ of $M$.

Next, for later use, we compute derivatives of principal curvatures (4.2) of the spherical projection (4.1). Let $X \in T_{p} M$ be a direction of curvature corresponding to the curvature sphere $\lambda k_{1}(p)+k_{2}(p)$, or equivalently to $\lambda A k_{1}(p)+$ $A k_{2}(p)$. From (4.1), we get

$$
\begin{aligned}
d \tilde{k}_{1}(X) & =X(\alpha) A k_{1}+X(\beta) A k_{2}+A\left(\alpha d k_{1}(X)+\beta d k_{2}(X)\right) \\
& \equiv(\alpha-\beta \lambda) A d k_{1}(X) \quad\left(\bmod \operatorname{span}\left(A k_{1}(p), A k_{2}(p)\right) .\right.
\end{aligned}
$$

So if $Y \in T_{p} M$ is also a direction of curvature corresponding to $\lambda k_{1}(p)+k_{2}(p)$, noting a) and c) in $\S 3$, we have

$$
\begin{aligned}
\langle d \boldsymbol{q}(X), d \boldsymbol{q}(Y)\rangle & =\left\langle d \tilde{k}_{1}(X), d \tilde{k}_{1}(Y)\right\rangle \\
& =(\alpha-\beta \lambda)^{2}\left\langle d k_{1}(X), d k_{2}(Y)\right\rangle \\
& =(\alpha-\beta \lambda)^{2}\langle d \boldsymbol{p}(X), d \boldsymbol{p}(Y)\rangle .
\end{aligned}
$$

Since it is easy to see that $\alpha-\beta \lambda=\frac{b \lambda+d}{a d-b c}$, we obtain:

LEMMA 4.9. If $X \in T_{p} M$ is a direction of the curvature sphere $\lambda k_{1}(p)+k_{2}(p)$ such that $\|d p(X)\|=1$, then $\frac{a d-b c}{b \lambda+d} d \boldsymbol{q}(X)$ is a unit principal vector corresponding to the principal curvature $\tilde{\lambda}=\frac{a \lambda+c}{b \lambda+d}$.

Now, let $U$ be a neighbourhood of $M$ on which principal curvatures satisfy $\lambda_{1}>\lambda_{2}>\cdots>\lambda_{g}$. Then the tangent bundle $T U$ is decomposed into $T U=T_{1}+\cdots$ $+T_{g}$ where $T_{2}$ is the curvature distribution corresponding to $\lambda_{2}$ and $\tilde{\lambda}_{2}$. We can choose a local frame $X_{1}, \cdots X_{n-1}$ of $T U$ so that $X_{m_{1}+\cdots+m_{\imath-1}+1}, \cdots, X_{m_{i}} \in T_{\imath}$, where $m_{\imath}$ is the multiplicitiy of $\lambda_{\imath}$, and that $e_{i}=d \boldsymbol{p}\left(X_{\imath}\right), i=1, \cdots, n-1$ form an orthonormal frame with respect to $p: M \rightarrow S^{n}$. Denoting the connection of $S^{n}$ by $\nabla$, we put

$$
\nabla_{e_{i}} e_{j}=\sum_{k=1}^{n-1} \Lambda_{i j}^{k} e_{k}+\lambda_{i} \delta_{i j} n
$$

Then we have [5]:

$$
e_{i}\left(\lambda_{j}\right)=\Lambda_{j j}^{2}\left(\lambda_{j}-\lambda_{\imath}\right), \quad i \neq j .
$$

Remark 4.10. Put $[i]:=\left\{j \mid \lambda_{2}=\lambda_{j}\right\}$. Then a Dupin hypersurface $\boldsymbol{p}: M \rightarrow S^{n}$ is isoparametric iff $\Lambda_{j j}^{i} \equiv 0$ for all $i, j$ such that $i \notin[j]$.

Since 


$$
f_{\imath}=\frac{a d-b c}{b \lambda_{i}+d} d \boldsymbol{q}\left(X_{\imath}\right),
$$

$i=1, \cdots, n-1$, form an orthnormal frame with respect to $q: M \rightarrow S^{n}$ by (4.3), putting

$$
\nabla_{f_{\imath}} f_{j}=\sum_{k=1}^{n-1} \tilde{\Lambda}_{\imath \jmath}^{k} f_{k}+\tilde{\lambda} \delta_{i j} \boldsymbol{m}
$$

we get

$$
f_{i}\left(\tilde{\lambda}_{j}\right)=\tilde{\Lambda}_{j j}^{2}\left(\tilde{\lambda}_{j}-\tilde{\lambda}_{\imath}\right), \quad i \neq j .
$$

For convenience, put $A=\left(\begin{array}{c}{ }^{t} b_{1} \\ \vdots \\ { }^{t} b_{n+3}\end{array}\right)=\left(\begin{array}{ccc}{ }^{t} c_{1} & & \\ \vdots & a_{n+2} & a_{n+3} \\ { }^{t} c_{n+3} & & \end{array}\right)$, where ${ }^{t} b_{\alpha}=\left(a_{\alpha \beta}\right)_{1 \leqq \beta \leqq n+3}$, ${ }^{t} c_{\alpha}=\left(a_{\alpha \imath}\right)_{1 \leqq \imath \Omega n+1}$ for $\alpha=1, \cdots, n+3, a_{n+2}=\left(a_{\alpha n+2}\right)_{1 \leqq \alpha \leqq n+3}$ and $a_{n+3}=\left(a_{\alpha n+3}\right)_{1 \leqq \alpha \leqq n+3}$.

LEMMA 4.11. We have

and for $i \notin[j]$,

$$
\tilde{\Lambda}_{\jmath \jmath}^{2}=0 \quad \text { if } \quad j \neq i \in[j],
$$

$$
\tilde{\Lambda}_{j j}^{\imath}=\frac{1}{b \lambda_{j}+d}\left\{\left(b \lambda_{j}+d\right) x_{i}-\left(a \lambda_{j}+c\right) y_{i}+(a d-b c) \Lambda_{j j}^{\imath}\right\}
$$

where $x_{\imath}=\left\langle c_{n+2}, e_{\imath}\right\rangle$ and $y_{\imath}=\left\langle c_{n+3}, e_{\imath}\right\rangle$, using the inner product $\left\langle\right.$, of $\boldsymbol{R}^{n+1}$.

Proof. Since $a=\left\langle c_{n+2}, \boldsymbol{p}\right\rangle+a_{n+2}{ }_{n+2}, \quad b=\left\langle c_{n+3}, \boldsymbol{p}\right\rangle+a_{n+3 n+2}, c=\left\langle c_{n+2}, \boldsymbol{n}\right\rangle+$ $a_{n+2 n+3}$ and $d=\left\langle c_{n+3}, \boldsymbol{n}\right\rangle+a_{n+3} n_{+3}$, it is easy to see that:

1) $e_{i}\left(a \lambda_{j}+c\right)=e_{i}(a) \lambda_{j}+e_{i}(c)=\left\langle c_{n+2}, \lambda_{j} e_{i}+\nabla_{e_{i}} n\right\rangle=0, \quad$ if $\quad j \neq \imath \in[j]$

2) $e_{i}\left(b \lambda_{j}+d\right)=\left\langle c_{n+3}, \lambda_{j} e_{i}+\nabla_{e_{i}} \boldsymbol{n}\right\rangle=0, \quad$ if $j \neq i \in[j]$

3) $e_{i}\left(a \lambda_{j}+c\right)=\left\langle c_{n+2}, \lambda_{j} e_{i}+\nabla_{e_{i}} n\right\rangle+a e_{i}\left(\lambda_{j}\right)$

$$
=\left(\lambda_{j}-\lambda_{\imath}\right)\left(\left\langle c_{n+2}, e_{\imath}\right\rangle+a \Lambda_{j j}^{2}\right), \quad \text { if } i \notin[j]
$$

4) $e_{i}\left(b \lambda_{j}+d\right)=\left(\lambda_{j}-\lambda_{\imath}\right)\left(\left\langle c_{n+3}, e_{\imath}\right\rangle+b \Lambda_{j j}^{2}\right), \quad$ if $i \notin[j]$.

So we have

and for $i \notin[j]$,

$$
e_{i}\left(\tilde{\lambda}_{j}\right)=0, \quad \text { if } \quad \jmath \neq i \in[j]
$$

$$
e_{i}\left(\tilde{\lambda}_{j}\right)=\frac{\left(\lambda_{j}-\lambda_{\imath}\right)}{\left(b \lambda_{j}+d\right)^{2}}\left\{\left(b \lambda_{j}+d\right) x_{\imath}-\left(a \lambda_{j}+c\right) y_{\imath}+(a d-b c) \Lambda_{j j}^{i}\right\} .
$$

Here we note that

and

$$
e_{2}\left(\lambda_{j}\right)=d \boldsymbol{p}\left(X_{2}\right)\left(\lambda_{j}\right)=X_{i}\left(\lambda_{j}\right)
$$

$$
e_{i}\left(\tilde{\lambda}_{j}\right)=X_{i}\left(\tilde{\lambda}_{j}\right)
$$


considering $\tilde{\lambda}$, as a function on $M$.

On the other hand, using (4.5), we get

$$
f_{i}\left(\tilde{\lambda}_{j}\right)=\frac{a d-b c}{b \lambda_{i}+d} d \boldsymbol{q}\left(X_{i}\right)\left(\tilde{\lambda}_{j}\right)=\frac{a d-b c}{b \lambda_{i}+d} X_{i}\left(\tilde{\lambda}_{j}\right)=\frac{a d-b c}{b \lambda_{i}+d} e_{i}\left(\tilde{\lambda}_{j}\right) .
$$

Thus we have

$$
e_{i}\left(\tilde{\lambda}_{j}\right)=\frac{b \lambda_{i}+d}{a d-b c} \tilde{\Lambda}_{j j}^{\imath}\left(\tilde{\lambda}_{j}-\tilde{\lambda}_{\imath}\right)=\tilde{\Lambda}_{\jmath j}^{\imath} \frac{\left(\lambda_{j}-\lambda_{\imath}\right)}{\left(b \lambda_{j}+d\right)},
$$

from which with (4.8) follows the lemma.

q.e.d.

\section{$\S 5$. Compact Dupin hypersurfaces with four principal curvatures.}

From now on, let $\boldsymbol{p}: M \rightarrow S^{n}$ be a compact connected embedded Dupin hypersurface with four principal curvatures $\lambda>\mu>\nu>\tau$. Then we know that $m_{1}=m_{3}$ and $m_{2}=m_{4}$, where $m_{1}, m_{2}, m_{3}, m_{4}$ are multiplicities of $\lambda, \mu, \nu, \tau$, respectively [7]. We use the index convention: $1 \leqq a, b, c \leqq m_{1}<i, j, k \leqq m_{1}+m_{2}<$ $r, s, t \leqq 2 m_{1}+m_{2}<u, v, w \leqq n-1$. A local orthonormal frame $\left(e_{a}, e_{\imath}, e_{r}, e_{u}\right)$ is chosen as in $\S 4$.

The purpose is now to find a condition under which there exists $A \in$ $O(n+1,2)$ such that $\tilde{\Lambda}_{\beta \beta}^{\alpha} \equiv 0$ for all $\alpha, \beta$ such that $\alpha \notin[\beta]$. But we come against difficulty soon if we investigate equations

$$
\tilde{\Lambda}_{\imath \imath}^{a}=0, \quad \tilde{\Lambda}_{r r}^{a}=0, \quad \tilde{\Lambda}_{u u}^{a}=0,
$$

even at a point, with $\left(x_{a}, y_{a}\right)$, defined in Lemma 4.11, as unknowns. At a critical point of $\Psi$, however, this can be done.

Proposition 5.1. Let $p$ be a critical point of $\Psi$. Then we obtain $A \in$ $O(n+1,2)$ such that

$$
\tilde{\Lambda}_{\beta \beta}^{\alpha}(p)=0, \quad \text { for all } \alpha, \beta \text { such that } \alpha \notin[\beta] \text {. }
$$

Remark 5.2. At $p$, the image hypersurface has the "common" normal geodesic defined in [5].

For the proof, we need:

LEMMA 5.3. At a critical point $p \in M$ of $\Psi$, we have:

(i ) $\frac{\Lambda_{\imath i}^{a}-\Lambda_{r r}^{a}}{\mu-\nu}=\frac{\Lambda_{i i}^{a}-\Lambda_{u u}^{a}}{\mu-\tau}=\frac{\Lambda_{r r}^{a}-\Lambda_{u u}^{a}}{\nu-\tau}\left(=: R_{a}\right)$

(ii) $\frac{\Lambda_{a a}^{\imath}-\Lambda_{r r}^{2}}{\lambda-\nu}=\frac{\Lambda_{a a}^{2}-\Lambda_{u u}^{2}}{\lambda-\tau}=\frac{\Lambda_{r r}^{2}-\Lambda_{u u}^{2}}{\nu-\tau}\left(=: R_{\imath}\right)$

(iii) $\frac{\Lambda_{a a}^{r}-\Lambda_{i i}^{r}}{\lambda-\mu}=\frac{\Lambda_{a a}^{r}-\Lambda_{u u}^{r}}{\lambda-\tau}=\frac{\Lambda_{i i}^{r}-\Lambda_{u u}^{r}}{\mu-\tau}\left(=: R_{r}\right)$ 
(iv) $\frac{\Lambda_{a a}^{u}-\Lambda_{i i}^{u}}{\lambda-\mu}=\frac{\Lambda_{a a}^{u}-\Lambda_{r r}^{u}}{\lambda-\nu}=\frac{\Lambda_{i i}^{u}-\Lambda_{r r}^{u}}{\mu-\nu}\left(=: R_{u}\right)$.

Proof. Let $\Phi=\log \Psi$. Then $p$ is a critical point of $\Psi$ iff $p$ is a critical point of $\Phi$. For instance, we have, using (4.4),

$$
\begin{aligned}
0=e_{a}(\Phi) & =\frac{e_{a}(\lambda-\mu)}{\lambda-\mu}-\frac{e_{a}(\lambda-\nu)}{\lambda-\nu}+\frac{e_{a}(\tau-\nu)}{\tau-\nu}-\frac{e_{a}(\tau-\mu)}{\tau-\mu} \\
& =\Lambda_{i i}^{a}-\Lambda_{r r}^{a}+\frac{\Lambda_{u u}^{a}(\tau-\lambda)-\Lambda_{r r}^{a}(\nu-\lambda)}{\tau-\nu}-\frac{\Lambda_{u u}^{a}(\tau-\lambda)-\Lambda_{i i}^{a}(\mu-\lambda)}{\tau-\mu} \\
& =(\lambda-\tau)\left\{\frac{\Lambda_{i i}^{a}-\Lambda_{u u}^{a}}{\mu-\tau}-\frac{\Lambda_{r r}^{a}-\Lambda_{u u}^{a}}{\nu-\tau}\right\} .
\end{aligned}
$$

The first equality in (i), then follows automatically. (ii) and (iii) are similarly proved.

q.e.d.

To prove Proposition 5.1, it is sufficient to show that there is a Lie transformation $A_{1} \in O(n+1,2)$ such that the image hypersurface has the "common" normal circle at $p[5]$, i. e. :

Lemma 5.4. Let $p$ be a critical point of $\Psi$. Then we obtain $A_{1} \in O(n+1,2)$ such that

$$
\tilde{\Lambda}_{\beta \beta}^{\alpha}(p)=\tilde{\Lambda}_{r r}^{\alpha}(p) \quad \text { for all } \alpha, \beta, \gamma \text { such that } \alpha \notin[\beta] \cup[\gamma], \beta \notin[\gamma] .
$$

Proof. Choose the coordinate of $\boldsymbol{R}^{n+1}$ so that $e_{\alpha}(p)={ }^{t}\left(0, \cdots \stackrel{\alpha}{1}^{\alpha} 0\right), \alpha=1, \cdots$, $n-1, \boldsymbol{p}(p)=^{t}(0, \cdots 1,0)$, and $\boldsymbol{n}(p)={ }^{t}(0, \cdots, 1)$. Consider simultaneous equations :

$$
\tilde{\Lambda}_{i \imath}^{a}(p)=\tilde{\Lambda}_{r r}^{a}(p)=\tilde{\Lambda}_{u u}^{a}(p)
$$

with unknowns $\left(x_{a}, y_{a}\right)$ for a fixed $a$, where we use notations in Lemma 4.11. For simplicity, try to solve (5.3) by putting $b=0$. Then from (4.7), (5.3) becomes

$$
\begin{aligned}
& a(\mu-\nu) y_{a}-a d\left(\Lambda_{i \imath}^{a}-\Lambda_{r r}^{a}\right)=0 \\
& a(\mu-\tau) y_{a}-a d\left(\Lambda_{\imath i}^{a}-\Lambda_{u u}^{a}\right)=0
\end{aligned}
$$

By (i) of Lemma 5.3, at $p$, a solution is, for instance,

$$
\left(x_{a}, y_{a}\right)=\left(0, d R_{a}\right), \quad a=1, \cdots, m_{1} .
$$

Just in the same way, solutions to

$$
\begin{aligned}
& \tilde{\Lambda}_{a a}^{\imath}(p)=\tilde{\Lambda}_{r r}^{\imath}(p)=\tilde{\Lambda}_{u u}^{\imath}(p), \\
& \tilde{\Lambda}_{a a}^{r}(p)=\tilde{\Lambda}_{i \imath}^{r}(p)=\tilde{\Lambda}_{u u}^{r}(p), \\
& \tilde{\Lambda}_{a a}^{u}(p)=\tilde{\Lambda}_{\imath i}^{u}(p)=\tilde{\Lambda}_{r r}^{u}(p),
\end{aligned}
$$


are, respectively,

$$
\begin{array}{ll}
\left(x_{\imath}, y_{\imath}\right)=\left(0, d R_{\imath}\right) & m_{1}<i \leqq m_{1}+m_{2}, \\
\left(x_{r}, y_{r}\right)=\left(0, d R_{r}\right) & m_{1}+m_{2}<r \leqq 2 m_{1}+m_{2}, \\
\left(x_{u}, y_{u}\right)=\left(0, d R_{u}\right) & 2 m_{1}+m_{2}<u \leqq n-1 .
\end{array}
$$

Now, we have to find a Lie frame $b_{1}, \cdots, b_{n+3}$ such that

$$
\begin{aligned}
& b_{n+2}={ }^{t}\left(x, x_{n}, x_{n+1}, a-x_{n}, c-x_{n+1}\right) \\
& b_{n+3}={ }^{t}\left(y, y_{n}, y_{n+1},-y_{n}, d-y_{n+1}\right),
\end{aligned}
$$

where $x=\left(x_{a}, x_{\imath}, x_{r}, x_{u}\right)$ and $y=\left(y_{a}, y_{\imath}, y_{r}, y_{u}\right)$ are given above, $x_{n}=\left\langle c_{n+2}, \boldsymbol{p}\right\rangle$, $x_{n+1}=\left\langle c_{n+2}, \boldsymbol{n}\right\rangle, y_{n}=\left\langle c_{n+3}, \boldsymbol{p}\right\rangle$ and $y_{n+1}=\left\langle c_{n+3}, \boldsymbol{n}\right\rangle$. Put $x_{n}=x_{n+1}=y_{n}=0, a=1$, $c=0$ and $y_{n+1}=-N d$. Then obviously, we have $\left\langle b_{n+2}, b_{n+2}\right\rangle=-1$ and $\left\langle b_{n+2}\right.$, $\left.b_{n+3}\right\rangle=0$. On the other hand, since

$$
\left\langle b_{n+3}, b_{n+3}\right\rangle=d^{2}\left\{\sum_{\alpha=1}^{n-1} R_{\alpha}^{2}+N^{2}-(N+1)^{2}\right\}=d^{2}\left\{\sum_{\alpha=1}^{n-1} R_{\alpha}^{2}-1-2 N\right\},
$$

we have $\left\langle b_{n+3}, b_{n+3}\right\rangle<0$ for a sufficiently large $N$, and then putting $d=$ ${\sqrt{\left|\sum_{\alpha=1}^{n-1} R_{\alpha}^{2}-1-2 N\right|}}^{-1}$, we get $\left\langle b_{n+3}, b_{n+3}\right\rangle=-1$. Therefore according to Remark 3.2 , we can extend $b_{n+2}, b_{n+3}$ to a Lie frame $b_{1}, \cdots, b_{n+3}$ so that $A_{1}=\left(\begin{array}{c}t \\ \vdots \\ \vdots \\ t b_{n+3}\end{array}\right) \in$ $O(n+1,2)$.

q.e.d.

Remark 5.5. The desired Lie transformation $A$ in Proposition is obtained now by $A=A_{2} \cdot A_{1}$, where $A_{2}$ is the conformal transformation of $S^{n}$ given by $A_{2}=\left(\begin{array}{c}{ }^{t} b_{1}^{\prime} \\ \vdots \\ { }^{t} b_{n+3}^{\prime}\end{array}\right) \in O(n+1,2) ; b_{1}^{\prime}, \cdots, b_{n+3}^{\prime}$ is a Lie frame extended from

$$
\begin{aligned}
& b_{n+2}^{\prime}={ }^{t}\left(x^{\prime},-a K, 0, a(K+1), 0\right), \\
& b_{n+3}^{\prime}={ }^{t}(0,0,0,0,1)
\end{aligned}
$$

where $x^{\prime}=\left(-a \tilde{\Lambda}_{i i}^{a}(p),-a \tilde{\Lambda}_{a a}^{2}(p),-a \tilde{\Lambda}_{a a}^{r}(p),-a \tilde{\Lambda}_{a a}^{u}(p)\right) \in \boldsymbol{R}^{n-1}, K$ and $a$ are chosen so that $\left\langle b_{n+2}^{\prime}, b_{n+2}^{\prime}\right\rangle=-1$.

\section{§ 6. Using tautness.}

We can start now from a compact embedded Dupin hypersurface $\boldsymbol{q}: M \rightarrow S^{n}$ which has a point $p \in M$, at which the normal geodesic is "common". The purpose of this section is to show:

Proposition 6.1. The normal geodesic $\gamma$ at $p$ cuts $M$ at eight points $p_{1}=$ 


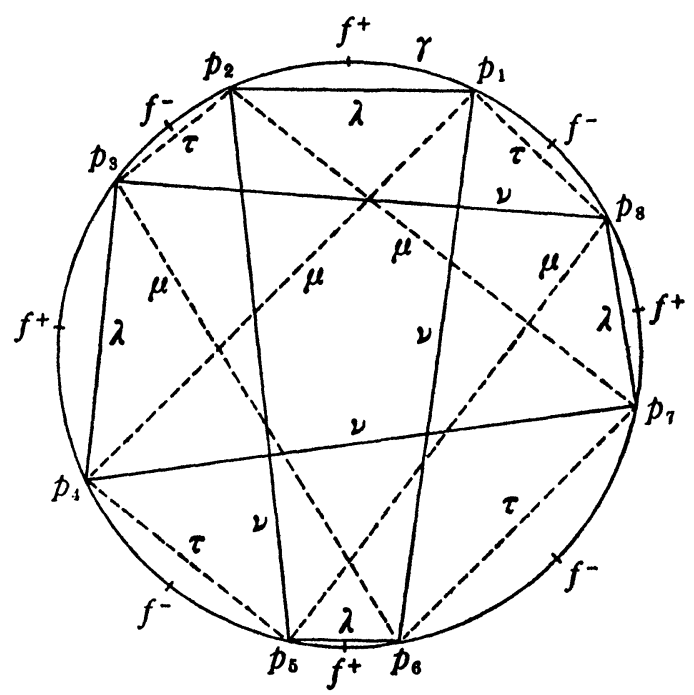

Fig. 6.1.

$p, p_{2}, \cdots, p_{8} . \quad \gamma$ is the common normal geodesic at every point $p_{i}(i=1, \cdots, 8)$. Moreover, all leaves at $p_{i}$ 's are connected as in Fig. 6.1.

Remark 6.2. The leaf $L_{p}^{\lambda}$ of a Dupin hypersurface $M$ corresponding to the principal curvature $\lambda$ at $p$ is an $m_{1}$-sphere centered at

where

$$
c=p+\frac{H_{a}}{\left\|H_{a}\right\|^{2}}
$$

$$
H_{a}=\sum_{\alpha \in[a]} \Lambda_{a a}^{\alpha} e_{\alpha}+\lambda \boldsymbol{n}-\boldsymbol{p}
$$

[5]. Obviously, $L_{p}^{\lambda}$ lies on the hypersphere $S_{p}^{\lambda}$ with center at

$$
f_{p}^{\lambda}=\cos \theta^{\lambda} p+\sin \theta^{\lambda} \boldsymbol{n},
$$

where $\theta^{\lambda}=\cot ^{-1} \lambda\left(-\frac{\pi}{2}<\theta^{\lambda} \leqq \frac{\pi}{2}\right)$ is its radius. With the normal geodesic $\gamma$ at $p, L_{p}^{\lambda}$ has another intersection $p^{\prime} \neq p$, iff $\Lambda_{a a}^{\alpha}(p)=0$ for all $\alpha \notin[a]$, that is if $L_{p}^{\lambda}$ is totally geodesic in $S_{p}^{\lambda}$. In this case, $\gamma$ is the normal geodesic at $p^{\prime}$, too [5, Remark 2.3].

Remark 6.3. By Thorbergsson [11], $H_{i}\left(M ; \boldsymbol{Z}_{2}\right) \neq 0$ only when $i=0, m_{1}, m_{2}$, $m_{1}+m_{2}, 2 m_{1}+m_{2}, m_{1}+2 m_{2}, n-1$ and $\beta\left(M ; Z_{2}\right)=8$. Non-zero cycles of $H_{m_{1}}(M)$ and $H_{m_{2}}(M)$ are represented by $L^{\lambda}$ and $L^{\tau}$, respectively. We denote other nontrivial cycles by 


$$
\begin{aligned}
& {\left[c^{\mu \lambda}\right] \in H_{m_{1}+m_{2}}(M),\left[c^{\nu \tau}\right] \in H_{m_{1}+m_{2}}(M),} \\
& {\left[c^{\nu \mu \lambda}\right] \in H_{2 m_{1}+m_{2}}(M),\left[c^{\mu \nu \tau}\right] \in H_{m_{1}+2 m_{2}}(M),}
\end{aligned}
$$

which are image cycles of the fundamental cycles of $N_{\imath}$ 's by maps $h_{\imath}$ 's defined in [11]. Note that the intersection number $S($, ) satisfies

$$
S\left(c^{\mu \lambda}, c^{\nu \tau}\right)=1, S\left(L^{\tau}, c^{\nu \mu \lambda}\right)=1, S\left(L^{\lambda}, c^{\mu \nu \tau}\right)=1 \text {. }
$$

We denote by $B_{p}^{\lambda_{ \pm}}$, the ball such that $\partial B_{p}^{\lambda_{ \pm}}=S_{p}^{\lambda}$ where $\boldsymbol{n}(p)$ is the inner (outer, resp.) normal to $B_{p}^{\lambda+}\left(B_{p}^{\lambda-}\right.$, resp.). $B_{p}^{\mu \pm}, B_{p}^{\nu \pm}$ and $B_{p}^{\tau \pm}$ are similarly defined.

LEMmA 6.4. For any $p \in M$ and any $L^{\lambda}\left(L^{\tau}\right.$, resp. $), B_{p}^{\mu-} \cap L^{\lambda} \neq \varnothing\left(B_{p}^{\nu+} \cap L^{\tau} \neq\right.$ $\varnothing$, resp.).

Proof. $B_{p}^{\mu-}$ contains the homology cycle $c^{\mu \nu \tau}$ with which any $m_{1}$-cycle $L^{\lambda}$ has the intersection number $\neq 0$.

q.e.d.

Proof of Proposition 6.1. At $p$, by (5.1) and by Remark 6.2, we have $\gamma \cap L_{p}^{\lambda}=\left\{p, p_{2}\right\}, \gamma \cap L_{p}^{\mu}=\left\{p, p_{4}\right\}, \gamma \cap L_{p}^{\nu}=\left\{p, p_{6}\right\}, \gamma \cap L_{p}^{\tau}=\left\{p, p_{8}\right\}$. We have $f_{p_{2}}^{\tau}$ $\left(f_{p_{4}}^{\lambda}\right.$, resp.) on $\gamma$ in the direction from $p_{2}$ to $p_{4}\left(p_{4}\right.$ to $p_{2}$, resp.), since along $L_{p}^{\lambda}$ and $L_{p}^{\mu}$, the positive normal direction is preserved. Moreover by tautness, we have $\left(B_{p_{2}}^{\tau}\right)^{\circ} \cap M=\varnothing$ and $\left(B_{p_{4}}^{\lambda}\right)^{\circ} \cap M=\varnothing$. Therefore $f_{p_{2}}^{\tau}$ and $f_{p_{4}}^{\lambda}$ are situated in this order along $\overparen{p_{2} p_{4}}$ from $p_{2}$ to $p_{4}$. On the other hand, by Thorbergsson [11], $S^{n}$ is decomposed into two disk bundles over two focal submanifolds $M_{ \pm}:=$ $\left\{f_{p}^{ \pm} \mid p \in M\right\}, f_{p}^{+}=f_{p}^{\lambda}, f_{p}^{-}=f_{p}^{\tau}$, both of which have $M$ as their boundaries. Thus there exists a point $p_{3} \in M$ between $f_{p_{2}}^{\tau}$ and $f_{p_{4}}^{\lambda}$ on $\overparen{p_{2} p_{4}}$. Similarly, there exist $p_{5}, p_{7} \in M$ on the arcs $\overparen{p_{4} p_{6}}, \overparen{p_{6} p_{8}}$, respectively.

Remark 6.5. At this stage, we say nothing about the relation among $p_{2}$, $p_{3}, p_{4}$. Neither do we know whether $M$ cuts $\gamma$ orthogonally at $p_{3}, p_{5}, p_{7}$, or not. The proof is completed by Lemma 6.6 and 6.7 .

In the following (and in $\S 9$ as well), we denote $L_{\imath}^{*}, S_{\imath}^{*}, B_{\imath}^{* \pm}$ for $L_{p_{i}}^{*}, S_{p_{i}}^{*}$, $B_{p}^{* \pm}$, respectively, for $i=1, \cdots, 8$ and $*=\lambda, \mu, \nu, \tau$.

LEMMA 6.6. $L_{i}^{\tau} \cap L_{\imath+2}^{\lambda}=p_{\imath+1}$, for $i=2,4,6$.

Proof. $[i=2]$. By tautness, we have $L_{2}^{\tau}=B_{2}^{\tau} \cap M \subset S_{2}^{\tau}$ and $L_{4}^{\lambda}=B_{4}^{\lambda} \cap M \subset S_{4}^{\lambda}$ [5, Lemma 3.5]. Then denoting here $\theta_{\imath}^{*}=\left|\theta_{\imath}^{*}\right|$, we have

$$
2 \theta_{2}^{\tau} \leqq d\left(p_{2}, p_{3}\right) \text { and } 2 \theta_{4}^{\lambda} \leqq d\left(p_{4}, p_{3}\right)
$$

where equalities imply $p_{3} \in L_{2}^{\tau}$ and $p_{3} \in L_{4}^{\lambda}$, respectively. Hence $L_{2}^{\tau} \cap L_{4}^{\lambda} \neq \varnothing$ implies $L_{2}^{\tau} \cap L_{4}^{\lambda}=\left\{p_{3}\right\}$. Now, suppose $L_{2}^{\tau} \cap L_{4}^{\lambda}=\varnothing$. Then 


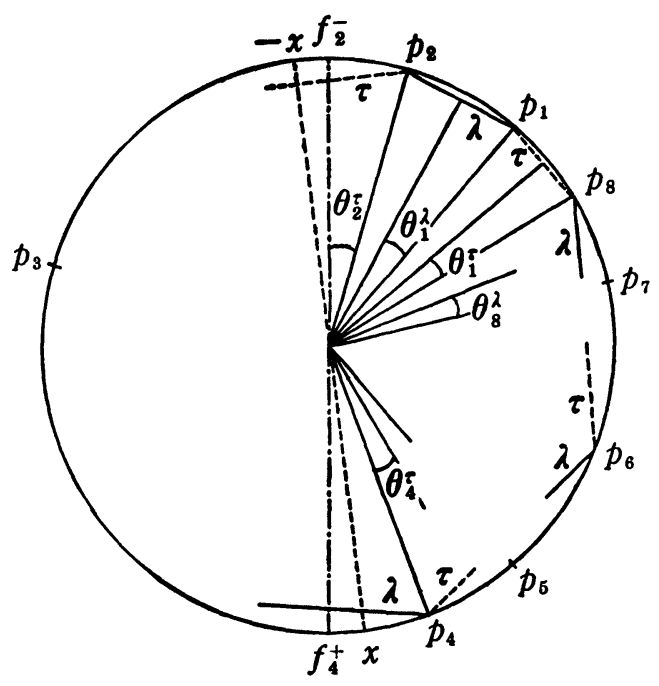

Fig. 6.2 .

$$
\theta_{2}^{\tau}<d\left(p_{3}, f_{2}^{-}\right), \quad \theta_{4}^{\lambda}<d\left(p_{3}, f_{4}^{+}\right) .
$$

Therefore we can transform $S^{n}$ conformally preserving $\gamma$ so that $S_{2}^{\tau}$ and $S_{4}^{\lambda}$ are centered at mutually antipodal points (Without confusion, we denote the image

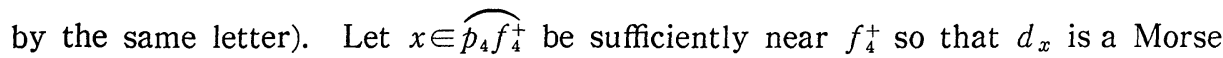
function on $M$. Let $B(x, r)$ be the ball centered at $x$ with radius $r$.

In $B\left(-x, d\left(-x, p_{2}\right)\right), d_{-x}$ has critical points of indicies 0 and $m_{2}$, the latter is nothing but $p_{2}$. Obviously as a critical point of $d_{-x}, p_{1}$ is of index $m_{1}$. Since

$$
d\left(p_{8}, f_{8}^{\nu}\right) \leqq \theta_{1}^{\tau}+\theta_{1}^{\lambda}+\theta_{2}^{\tau}
$$

by Lemma 6.4 , the index of $p_{8}$ as a critical point of $d_{-x} \geqq m_{1}+m_{2}$. In the same way, the index of $p_{8}$ as a critical point of $d_{x} \geqq m_{1}+m_{2}$. Thus the index of $p_{8}$ with respect to $d_{-x}$ is $m_{1}+m_{2}$, corresponding to the cycle $c^{\nu \tau}$. On the other hand, since $B_{2}^{\mu+}$ contains $c^{\mu \lambda}$ which is not homotopic to $c^{\nu \tau}$, and since $d\left(f_{2}^{\mu}, p_{2}\right)$ $\leqq \theta_{1}^{\lambda}+\theta_{1}^{\tau}+\theta_{8}^{\lambda}$ by Lemma $6.4, d_{-x}$ should have another critical point of index $m_{1}+m_{2}$ in $B:=B\left(-x, d\left(-x, p_{8}\right)+2 \theta_{8}^{\lambda}\right)$. Now, in $B\left(x, d\left(x, p_{4}\right)+2 \theta_{4}^{\tau}\right), d_{x}$ has three critical points corresponding to a point, $L_{4}^{\lambda}$ and $L_{4}^{\tau}$. (Note that we may assume $\left.d\left(x, f_{4}^{+}\right)<\theta_{4}^{\tau}\right)$ Thus from $p_{6} \in B^{c}, d_{x}$ has four critical points in $B^{c}$. Finally, $d_{-x}$ should have nine critical points on $M$, which contradicts tautness. $[i=6]$. Similar to $[i=2]$.

$[i=4]$. Easier than above two cases.

q.e.d.

LEMMA 6.7. $L_{2}^{\mu}=L_{7}^{\mu}, L_{3}^{\mu}=L_{6}^{\mu}, L_{5}^{\mu}=L_{8}^{\mu}$,

$$
L_{2}^{\nu}=L_{5}^{\nu}, L_{3}^{\nu}=L_{8}^{\nu}, L_{4}^{\nu}=L_{7}^{\nu} \text {. }
$$


Proof. $\left[L_{2}^{\mu}=L_{7}^{\mu}\right]$. Consider the cycle $c_{2}^{\mu \lambda} \subset B_{2}^{\mu+}$ at $p_{2}$ and $c_{7}^{\nu \tau} \subset B_{7}^{\nu-}$ at $p_{7}$. Then $S\left(c_{2}^{\mu \lambda}, c_{7}^{\nu \tau}\right)=1$ and $S\left(c_{2}^{\mu \nu \tau}, L_{7}^{\lambda}\right)=1$ imply $B_{2}^{\mu+} \cap B_{7}^{\nu-}=\left\{p_{7}\right\}$ and $p_{7} \in c_{2}^{\mu \lambda}$. Since $c_{2}^{\mu \lambda} \cap S_{2}^{\mu \lambda}=L_{2}^{\mu}$, we get $p_{7} \in L_{2}^{\mu}$, that is $L_{2}^{\mu}=L_{7}^{\mu}$. The other cases follow similarly.

q.e.d.

LEMMA 6.8. Let $z_{i}$ be the complex number corresponding to $p_{i} \in S^{1} \subset \boldsymbol{R}^{2} \cong \boldsymbol{C}$, $i=1, \cdots, 8$. Then we have

$$
\Psi\left(p_{1}\right)=\left[z_{2}, z_{8} ; z_{4}, z_{6}\right],
$$

where $\left[z_{2}, z_{8} ; z_{4}, z_{6}\right]=\frac{z_{2}-z_{4}}{z_{2}-z_{6}} / \frac{z_{8}-z_{4}}{z_{8}-z_{6}}$ is the cross ratio. $\Psi\left(p_{\imath}\right), i=2, \cdots, 8$ are similarly obtained.

Proof. As is well-known,

$$
\begin{aligned}
{\left[z_{1},-z_{1} ; z_{2}, z_{4}\right] } & =\tan \frac{1}{2}\left\{\arg \left(z_{2} / z_{1}\right)\right\} / \tan \frac{1}{2}\left\{\arg \left(z_{4} / z_{1}\right)\right\} \\
& =\frac{\mu\left(p_{1}\right)}{\lambda\left(p_{1}\right)} .
\end{aligned}
$$

Therefore

$$
\begin{aligned}
\Psi\left(p_{1}\right)=\frac{1-\mu / \lambda}{1-\nu / \lambda} / \frac{1-\mu / \tau}{1-\nu / \tau} & =\frac{1-\left[z_{1},-z_{1} ; z_{2}, z_{4}\right]}{1-\left[z_{1},-z_{1} ; z_{2}, z_{6}\right]} / \frac{1-\left[z_{1},-z_{1} ; z_{8}, z_{4}\right]}{1-\left[z_{1},-z_{1} ; z_{8}, z_{6}\right]} \\
& =\left[z_{2}, z_{8} ; z_{4}, z_{6}\right] . \quad \text { q.e.d. }
\end{aligned}
$$

\section{§7. Lie transformation of $S^{1}$.}

In this section, we find a Lie transformation $A_{3} \in O(2,2) \subset O(n+1,2)$, which maps $\gamma \cap M$ from the position in Fig. 6.1 to the position in Fig. 7.2. We follow the argument in [5]. That is, we restrict our argument to $\gamma=S^{1}$, on which, any pair of points connected by some leaf is considered as an oriented hypersphere of $S^{1}$. We give positive orientation to a leaf $L_{i}^{*}$ if $\theta_{i}^{*}=\cot ^{-1} *_{i}>0, *=$ $\lambda, \mu, \nu, \tau$. (Recall that $\theta_{\imath}^{*}$ is chosen so that $-\pi / 2<\theta_{\imath}^{*} \leqq \pi / 2$ ).

First of all, since $L_{1}^{\lambda} \cap L_{5}^{\lambda}=\{\phi\}$, we can transform $S^{1}$ conformally so that the images of $L_{1}^{\lambda}$ and $L_{5}^{\lambda}$ are centered at mutually antipodal points. Then by a conformal transformation fixing these centers, we may assume that $\lambda\left(p_{1}\right)=$ $\cot \theta=\lambda\left(p_{5}\right)$ for some $0<\theta<\pi / 2$. Note that in $\S 9$, we denote the combination of these conformal transformations by $C$.

Now, put $\varphi=\cot ^{-1}\left(-\tau\left(p_{2}\right)\right), \phi=\cot ^{-1}\left(-\tau\left(p_{1}\right)\right), \varphi^{\prime}=\cot ^{-1}\left(-\tau\left(p_{4}\right)\right)$ and $\phi^{\prime}=$ $\cot ^{-1}\left(-\tau\left(p_{6}\right)\right)$, where $0<\varphi, \phi, \varphi^{\prime}, \phi^{\prime}<\pi / 2$ (see Fig. 7.1). Then since $(\theta+\varphi+\phi)+$ $\left(\theta+\varphi^{\prime}+\psi^{\prime}\right)$ is less than $\pi$, we may assume

$$
\theta+\varphi+\phi<\frac{\pi}{2},
$$




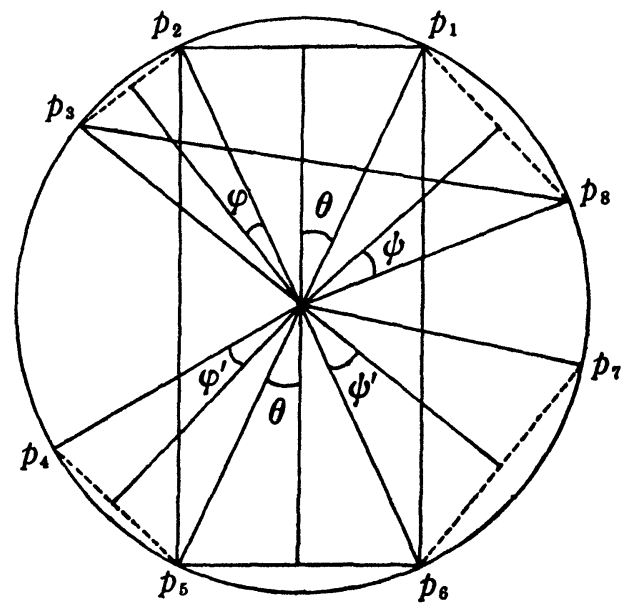

Fig. 7.1.

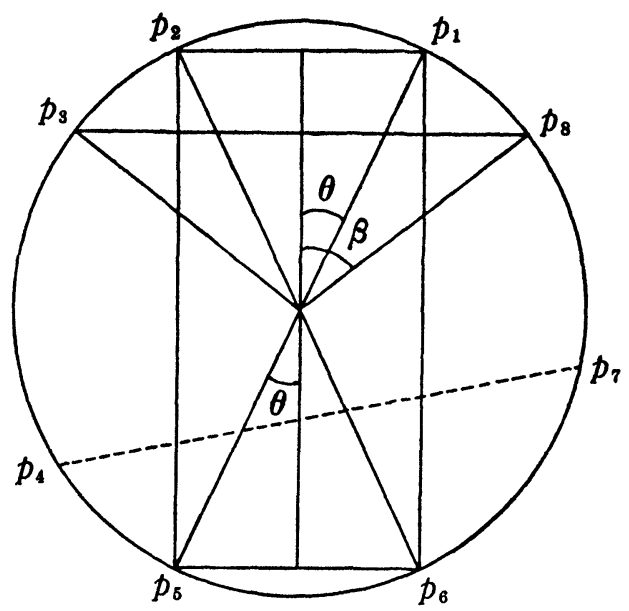

Fig. 7.2

without loss of generality. Let

$$
\begin{aligned}
& k_{1}=\left\{p_{1}, p_{2}\right\}={ }^{t}\left(\frac{1}{\cos \theta}, 0,1, \tan \theta\right), \\
& k_{2}=\left\{p_{5}, p_{6}\right\}={ }^{t}\left(-\frac{1}{\cos \theta}, 0,1, \tan \theta\right) \\
& k_{3}=\left\{p_{3}, p_{8}\right\}={ }^{t}\left(\frac{\cos \omega}{\cos \sigma}, \frac{\sin \omega}{\cos \sigma}, 1,-\tan \sigma\right),
\end{aligned}
$$


where $\sigma=\theta+\varphi+\phi, \omega=\phi-\varphi$, and

$$
k_{4}={ }^{t}(0,0,0,1) \text {. }
$$

Note that $k_{1}, k_{2}, k_{3} \in Q$ but $k_{4} \notin Q$. Moreover, let

$$
\begin{aligned}
& h_{1}=k_{1}, \\
& h_{2}=u k_{2}, \quad u \in \boldsymbol{R}-\{0\}, \\
& h_{3}=v^{t}\left(\frac{1}{\cos \beta}, 0,1,-\tan \beta\right), \quad v \in \boldsymbol{R}-\{0\}, \\
& h_{4}={ }^{t}(x, y, z, w) .
\end{aligned}
$$

Note that $h_{1}, h_{2}, h_{3} \in Q$. For $K=\left(k_{1}, k_{2}, k_{3}, k_{4}\right)$ and $H=\left(h_{1}, h_{2}, h_{3}, h_{4}\right)$, find $A_{3} \in$ $O(2,2)$ such that $A_{3} K=H$. Since

$$
K^{-1}=\left(\begin{array}{cccc}
\frac{\cos \theta}{2} & -\frac{\cos \sigma}{2 \sin \omega}\left(1+\frac{\cos \theta \cos \omega}{\cos \sigma}\right) & \frac{1}{2} & 0 \\
-\frac{\cos \theta}{2} & -\frac{\cos \sigma}{2 \sin \omega}\left(1-\frac{\cos \theta \cos \omega}{\cos \sigma}\right) & \frac{1}{2} & 0 \\
0 & \frac{\cos \sigma}{\sin \omega} & 0 & 0 \\
0 & \frac{\cos \sigma}{\sin \omega}(\tan \sigma+\tan \theta) & -\tan \theta & 1
\end{array}\right)
$$

$A_{3}=H K^{-1}=\left(a_{1}, a_{2}, a_{3}, a_{4}\right)$ is given by

$$
\begin{aligned}
& a_{1}=\frac{\cos \theta}{2}\left(h_{1}-h_{2}\right), \\
& a_{2}=\frac{\cos \sigma}{\sin \omega}\left(-\frac{\cos \omega}{\cos \sigma} a_{1}-a_{3}+h_{3}+\tan \sigma h_{4}\right), \\
& a_{3}=\frac{1}{2}\left(h_{1}+h_{2}\right)-\tan \theta h_{4}, \\
& a_{4}=h_{4} .
\end{aligned}
$$

By writing down the condition $A_{3} \in 0(2,2)$, i. e.

$$
\begin{aligned}
& \left\langle a_{1}, a_{1}\right\rangle=\left\langle a_{2}, a_{2}\right\rangle=-\left\langle a_{3}, a_{3}\right\rangle=-\left\langle a_{4}, a_{4}\right\rangle=1, \\
& \left\langle a_{\imath}, a_{\jmath}\right\rangle=0 \text { for } i \neq j,
\end{aligned}
$$

as equations with unknowns $(u, v, \beta, x, y, z, w)$, we have

$$
u=1
$$




$$
\begin{aligned}
& v=\frac{\cos \omega \cos \beta}{\cos \sigma} \\
& \cos (\theta+\beta)=\frac{\cos (\theta+\sigma)}{\cos \omega} \\
& x=0 \\
& z+w \tan \theta=\tan \theta \\
& -z+w \tan \beta=\frac{\sin \sigma}{\cos \omega \cos \beta} \\
& y^{2}-z^{2}-w^{2}=-1 .
\end{aligned}
$$

In fact,

(1) $1=\left\langle a_{1}, a_{1}\right\rangle$ implies (7.2) and $a_{1}=^{t}(1,0,0,0), 1 / 2\left(h_{1}+h_{2}\right)={ }^{t}(0,0,1, \tan \theta)$.

(2) $0=\left\langle a_{1}, a_{4}\right\rangle$ implies (7.5).

(3) $\left\langle a_{1}, a_{3}\right\rangle=0$ follows from (1) and (2).

(4) $0=\left\langle a_{1}, a_{2}\right\rangle=-\frac{\cos \omega}{\sin \omega}+\frac{\cos \sigma}{\sin \omega}\left\langle a_{1}, h_{3}\right\rangle$ implies $\left\langle a_{1}, h_{3}\right\rangle=\frac{\cos \omega}{\cos \sigma}$ and (7.3).

(5) $0=\left\langle a_{3}, a_{4}\right\rangle$ implies (7.6).

(6) $\left\langle a_{3}, a_{3}\right\rangle=-1-\tan ^{2} \theta-\tan ^{2} \theta-2 \tan \theta(-z-w \tan \theta)=-1$ follows from (5).

(7) $0=\left\langle a_{2}, a_{4}\right\rangle=\frac{\cos \sigma}{\sin \omega}\left(\left\langle h_{3}, h_{4}\right\rangle-\tan \sigma\right)$ implies $\left\langle h_{3}, h_{4}\right\rangle=\tan \sigma:(7.7)$.

(8) $0=\left\langle a_{2}, a_{3}\right\rangle=\frac{\cos \sigma}{\sin \omega}\left(1+\left\langle h_{3}, a_{3}\right\rangle\right)$ implies $\left\langle a_{3}, h_{3}\right\rangle=-1$ so that $v(-1+$ $\tan \theta \tan \beta)-\tan \theta \tan \sigma=-1$, i. e. (7.4).

(9) $\left\langle a_{2}, a_{2}\right\rangle=\frac{\cos ^{2} \sigma}{\sin ^{2} \omega}\left(\frac{\cos ^{2} \omega}{\cos ^{2} \sigma}-1-\tan ^{2} \sigma-2 \frac{\cos \omega}{\cos \sigma}\left\langle a_{1}, h_{3}\right\rangle\right.$

$$
\begin{aligned}
& \left.-2\left\langle a_{3}, h_{3}\right\rangle+2 \tan \sigma\left\langle h_{3}, h_{4}\right\rangle\right) \\
= & 1
\end{aligned}
$$

follows from (4), (7) and (8).

(10) $-1=\left\langle a_{4}, a_{4}\right\rangle$ implies (7.8).

Case 1. If $\theta+\sigma=2 \theta+\varphi+\phi \leqq \pi / 2$ is satisfied, then since

$$
0<\varphi \sim \phi<\varphi+\phi<2 \theta+\varphi+\phi=\theta+\sigma \leqq \frac{\pi}{2},
$$

we get

and so

$$
\cos \omega=\cos (\psi-\varphi)>\cos (\theta+\sigma) \geqq 0,
$$

$$
0 \leqq \frac{\cos (\theta+\sigma)}{\cos \omega}<1
$$


Thus we can find $\beta$ satisfying (7.4). Then, $v$ is obtained by (7.3). On the other hand, we get

$$
w=\frac{1}{\sin (\theta+\beta)}\left\{\sin \theta \cos \beta+\frac{\sin \sigma}{\cos \omega} \cos \theta\right\}
$$

from (7.6) and (7.7), so it follows

$$
w-1=\frac{\cos \theta}{\sin (\theta+\beta)}\left\{\frac{\sin \sigma}{\cos \omega}-\sin \beta\right\} .
$$

Now,

$$
\cos (\theta+\beta)=\frac{\cos (\theta+\sigma)}{\cos \omega} \geqq \cos (\theta+\sigma)
$$

implies $\beta \leqq \sigma$ because of $\theta+\sigma \leqq \pi / 2$, and hence we have

$$
\sin \beta \leqq \sin \sigma \leqq \frac{\sin \sigma}{\cos \omega},
$$

i.e. $w \geqq 1$. This guarantees $y^{2} \geqq 0$ in (7.8), and finally, the whole solution is given by

$$
\left\{\begin{array}{l}
u=1, v=\frac{\cos \omega \cos \beta}{\cos \sigma} \text { where } \beta=\cos ^{-1}\left\{\frac{\cos (\theta+\sigma)}{\cos \omega}\right\}-\theta, \\
x=0, y=\sqrt{z^{2}+w^{2}-1}, \text { where } z=\frac{\sin \theta}{\sin (\theta+\beta)}\left\{\sin \beta-\frac{\sin \sigma}{\cos \omega}\right\}, \\
w=\frac{1}{\sin (\theta+\beta)}\left\{\sin \theta \cos \beta+\frac{\sin \sigma}{\cos \omega} \cos \theta\right\} .
\end{array}\right.
$$

Case 2. When $\theta+\sigma=2 \theta+\varphi+\phi>\pi / 2$, we have

$$
0<\varphi \sim \phi<\pi-2 \theta-\varphi-\phi=\pi-\theta-\sigma<\frac{\pi}{2}
$$

since $2(\theta+\varphi)<\pi$ and $2(\theta+\phi)<\pi$ by (7.1). This implies

$$
\cos \omega>\cos (\pi-\theta-\sigma)=-\cos (\theta+\sigma)>0,
$$

and we get $\beta$ satisfying (7.4), $0<\beta<\pi$. On the other hand, from

$$
\cos (\theta+\beta)=\frac{\cos (\theta+\sigma)}{\cos \omega} \leqq \cos (\theta+\sigma)<0,
$$

noting that $\theta+\sigma<\pi$, we have $\beta \geqq \sigma$. Thus we obtain, using (7.1),

$$
\cos \beta \leqq \cos \sigma \leqq \frac{\cos \sigma}{\cos \omega} .
$$

Now, since (7.4) is equal to

$$
\cos \theta \cos \beta-\sin \theta \sin \beta=\frac{\cos \theta \cos \sigma-\sin \theta \sin \sigma}{\cos \omega},
$$


we get

$$
\sin \beta \leqq \frac{\sin \sigma}{\cos \omega},
$$

equivalently $w \geqq 1$. Thus, in this case again, solutions are given by (7.9).

\section{§ 8. Under the assumption that $\Psi$ is constant on $M$.}

Here, for the first time, we assume that $\Psi$ is constant on $M$.

Proposition 8.1. If $\Psi$ is constant on $M$, then there is a Lie transformation $A_{4} \in O(2,2)$ which maps $\gamma \cap M$ in Fig. 7.2 to the position in Fig. 8.1.

Proof. Consider the image hypersurface shown in Fig. 7.2. Note that $\nu\left(p_{1}\right)=\nu\left(p_{2}\right)$ and $\tau\left(p_{1}\right)=\tau\left(p_{2}\right)$ imply $\mu\left(p_{1}\right)=\mu\left(p_{2}\right)$. Now, let $\eta=\cot ^{-1} \lambda\left(p_{3}\right), \varphi=$ $\cot ^{-1}\left(-\tau\left(p_{2}\right)\right)$ and $\varphi^{\prime}=\cot ^{-1}\left(-\tau\left(p_{4}\right)\right)$, where $0<\eta, \varphi, \varphi^{\prime}<\pi / 2$. Without loss of generality, assume that $\varphi \leqq \varphi^{\prime}$. Then we have

$$
\theta+2 \varphi+\eta \leqq \frac{\pi}{2}
$$

To imitate the previous calculation, rotating the coordinate axes by $\pi / 2$, we put

$$
\begin{aligned}
& k_{1}=\left\{p_{2}, p_{5}\right\}={ }^{t}\left(\frac{1}{\sin \theta}, 0,1,-\cot \theta\right), \\
& k_{2}=\left\{p_{1}, p_{6}\right\}={ }^{t}\left(-\frac{1}{\sin \theta}, 0,1,-\cot \theta\right),
\end{aligned}
$$

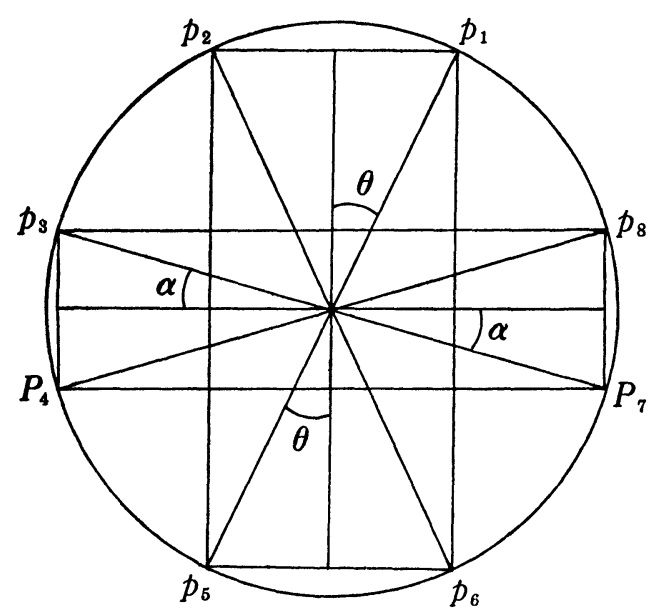

Fig. 8.1. 


$$
\begin{aligned}
& k_{3}=\left\{p_{3}, p_{4}\right\}={ }^{t}\left(\frac{\sin \zeta}{\cos \eta}, \frac{\cos \zeta}{\cos \eta}, 1, \tan \eta\right), \zeta=\theta+2 \varphi+\eta, \\
& k_{4}={ }^{t}(0,0,0,1), \\
& h_{1}=k_{1}, \\
& h_{2}=u k_{2}, \quad u \in \boldsymbol{R}-\{0\}, \\
& h_{3}=v^{t}\left(\frac{1}{\cos \alpha}, 0,1, \tan \alpha\right), \quad v \in \boldsymbol{R}-\{0\}, \\
& h_{4}={ }^{t}(x, y, z, w) .
\end{aligned}
$$

Now, find $A_{4} \in O(2,2)$ such that $A_{4} K=H$, where $K=\left(k_{1}, k_{2}, k_{3}, k_{4}\right)$ and $H=$ $\left(h_{1}, h_{2}, h_{3}, h_{4}\right)$. As before, since we have

$$
K^{-1}=\left(\begin{array}{cccc}
\frac{\sin \theta}{2} & -\frac{\cos \eta}{2 \cos \zeta}\left(1+\frac{\sin \zeta \sin \theta}{\cos \eta}\right) & \frac{1}{2} & 0 \\
-\frac{\sin \theta}{2} & -\frac{\cos \eta}{2 \cos \zeta}\left(1-\frac{\sin \zeta \sin \theta}{\cos \eta}\right) & \frac{1}{2} & 0 \\
0 & \frac{\cos \eta}{\cos \zeta} & 0 & 0 \\
0 & -\frac{\cos \eta}{\cos \zeta}(\tan \eta+\tan \theta) & \cot \theta & 1
\end{array}\right),
$$

$A_{4}$ is given by

$$
\begin{aligned}
& a_{1}=\frac{\sin \theta}{2}\left(h_{1}-h_{2}\right), \\
& a_{2}=\frac{\cos \eta}{\cos \zeta}\left\{-\frac{\sin \zeta}{\cos \eta} a_{1}-a_{3}+h_{3}-\tan \eta h_{4}\right\}, \\
& a_{3}=\frac{1}{2}\left(h_{1}+h_{2}\right)+\cot \theta h_{4}, \\
& a_{4}=h_{4} .
\end{aligned}
$$

The condition $A_{4} \in O(2,2)$ are, this time:

$$
\begin{aligned}
& u=1 \\
& \sin (\theta-\alpha)=\frac{\sin (\theta-\eta)}{\sin \zeta} \\
& v=\frac{\cos \alpha \sin \zeta}{\cos \eta} \\
& x=0
\end{aligned}
$$




$$
\begin{aligned}
& -z+w \cot \theta=\cot \theta \\
& z+w \tan \alpha=\frac{\sin \eta}{\cos \alpha \sin \zeta} \\
& y^{2}-z^{2}-w^{2}=-1 .
\end{aligned}
$$

As before, from (8.1) and

$$
\theta \sim \eta<\theta+\eta<\theta+2 \varphi+\eta=\zeta,
$$

there exists $\alpha$ satisfying (8.3). From (8.6) and (8.7), we get

and

$$
w=\frac{1}{\cos (\theta-\alpha)}\left(\cos \theta \cos \alpha+\frac{\sin \theta \sin \eta}{\sin \zeta}\right)
$$

$$
w-1=\frac{\sin \theta}{\cos (\theta-\alpha)}\left(\frac{\sin \eta}{\sin \zeta}-\sin \alpha\right) .
$$

When $\theta \geqq \eta$, it follows $\alpha \leqq \eta$ from (8.3) so that

implies $w \geqq 1$, and hence $y^{2} \geqq 0$.

$$
\sin \alpha \leqq \sin \eta \leqq \frac{\sin \eta}{\sin \zeta}
$$

When $\theta<\eta$, though $\alpha>\eta$, we have from (8.3),

$$
\begin{aligned}
\frac{\sin \theta \cos \eta-\cos \theta \sin \eta}{\sin \zeta} & =\sin \theta \cos \alpha-\cos \theta \sin \alpha \\
& <\sin \theta \cos \eta-\cos \theta \sin \alpha \\
& \leqq \sin \theta \frac{\cos \eta}{\sin \zeta}-\cos \theta \sin \alpha
\end{aligned}
$$

so that

$$
\frac{\sin \eta}{\sin \zeta}>\sin \alpha
$$

Therefore in both cases, solutions are given by

$$
\left\{\begin{array}{l}
u=1, \quad \alpha=\theta-\sin ^{-1}\left\{\frac{\sin (\theta-\eta)}{\sin \zeta}\right\}, \quad v=\frac{\cos \alpha \sin \zeta}{\cos \eta}, \\
x=0, \quad y=\sqrt{z^{2}+w^{2}-1,} \text { where } z=\frac{\cos \theta}{\cos (\theta-\alpha)}\left(\frac{\sin \eta}{\sin \zeta}-\sin \alpha\right), \\
w=\frac{1}{\cos (\theta-\alpha)}\left(\cos \theta \cos \alpha+\frac{\sin \theta \sin \eta}{\sin \zeta}\right) .
\end{array}\right.
$$

Finally, by using $\Psi$ is constant, we get $\tau\left(p_{2}\right)=\tau\left(p_{4}\right) \stackrel{(*)}{=} \tau\left(p_{6}\right)=\tau\left(p_{8}\right)$. Put $\rho=$

$\left(^{*}\right)$ By an easy calculation, we have for $k_{5}=\left\{p_{5}, p_{7}\right\}, A k_{5}={ }^{t}\left(-\frac{1}{\cos \alpha}, 0,1, \tan \alpha\right)$, and the equality holds. 
$\cot ^{-1}\left(-\tau\left(p_{2}\right)\right)$

Proposition 8.2. If $\Psi$ is constant on $M$, then we have $\theta+\rho=\pi / 4$ and $\theta=\alpha$.

Proof. By Lemma 6.8, we have

$$
\begin{aligned}
& \Psi\left(p_{1}\right)=\frac{z_{2}-z_{4}}{z_{2}-z_{6}} / \frac{z_{8}-z_{4}}{z_{8}-z_{6}}=\frac{e^{2 \imath(\alpha+\rho)}-1}{-2} \cdot \frac{e^{-2 \imath(\alpha+\rho)}-1}{-2} \\
& \Psi\left(p_{4}\right)=\frac{z_{3}-z_{1}}{z_{3}-z_{7}} / \frac{z_{5}-z_{1}}{z_{5}-z_{7}}=\frac{e^{-2 \imath(\alpha+\rho)}-1}{-2} \cdot \frac{e^{2 \imath(\theta+\rho)}-1}{-2} .
\end{aligned}
$$

From $(\alpha+\rho)+(\theta+\rho)=\pi / 2$, it follows

$$
e^{2 \imath(\theta+\rho)}=e^{2(\pi-2(\alpha+\rho))}=-e^{-2 \imath(\alpha+\rho)} .
$$

So if we put $z=e^{2 \imath(\theta+\rho)}, \Psi\left(p_{1}\right)=\Psi\left(p_{4}\right)$ is written as

Thus we get

$$
(z-1)(\bar{z}-1)=(z+1)(\bar{z}+1) \text {. }
$$

$$
\theta+\rho=\alpha+\rho=\frac{\pi}{4} .
$$

q.e.d.

COROLlARY 8.3. If $\Psi$ is constant on $M$, then by taking $A_{5} \in O(2,2)$ of type (3.5), we can transform Fig. 8.1 so that $\theta=\alpha=\rho=\pi / 8$. Therefore, in fact, $\Psi=1 / 2$.

\section{§9. Proof of Theorem II.}

From the results in $\S 5-8$, when $\Psi$ is constant on $M$, we see that at each point $p$ of $M$, there exists Lie transformation $A_{p}=A_{5} \cdot A_{4} \cdot A_{3} \cdot C \cdot A_{2} \cdot A_{1}$ of which image satisfies the relation in Fig. 9.1 where we denote the image point of $p$ by $p_{1}$ and the normal geodesic at $p_{1}$ by $\gamma$. Note that this result is obtained not only by a local calculation but also by using a global property "tautness" of $M$.

Now we show:

Proposition 9.1. Let $N$ be a Lie image of an isoparametric hypersurface $N_{0}$ with four principal curvatures. Assume that at some point $p_{1} \in N$, the intersection of $N$ with the normal geodesic $\gamma$ at $p_{1}$ makes a regular octagon $p_{1} p_{2} \ldots$ $p_{8}$ as in Fig. 9.1, where $\gamma$ is common at each $p_{i}$. Then $N$ is itself isoparametric.

Proof. By a parallel transformation, if necessary, we may assume that $\lambda \equiv \cot (\pi / 8) \equiv-\tau$ and $\mu \equiv \cot (3 \pi / 8) \equiv-\nu$ on $N_{0}$. Let $N$ be the image of $N_{0}$ by some $A \in O(n+1,2)$. Let $q_{\imath}$ be the inverse image point of $p_{2}$. Then $A$ maps the regular octagon at $q_{1}$ to the regular octagon at $p_{1}$. This means, up to isometry, that if we put 


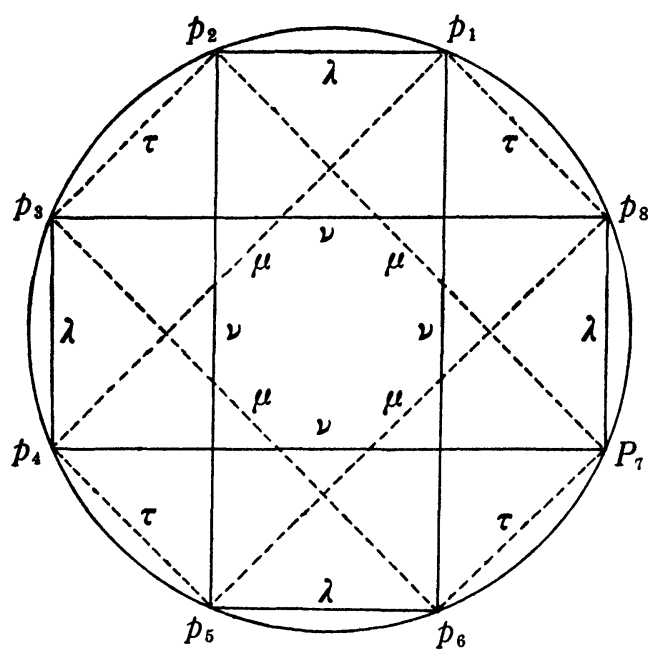

Fig. 9.1.

$$
\begin{aligned}
& k_{1}\left(q_{\imath}\right)=k_{1}\left(p_{\imath}\right)={ }^{t}\left(0, \cos \frac{(i-1)}{4} \pi, \sin \frac{(i-1)}{4} \pi, 1,0\right) \\
& k_{2}\left(q_{i}\right)=k_{2}\left(p_{\imath}\right)={ }^{t}\left(0,(-1)^{\imath} \sin \frac{(i-1)}{4} \pi,(-1)^{\imath-1} \cos \frac{(i-1)}{4} \pi, 0,1\right)
\end{aligned}
$$

for $i=1,2, \cdots, 8$, and $0=(0, \underset{n-1}{,}, 0)$, then

where

$$
\begin{aligned}
& \alpha\left(q_{\imath}\right) A k_{1}\left(q_{i}\right)+\beta\left(q_{\imath}\right) A k_{2}\left(q_{\imath}\right)=k_{1}\left(p_{\imath}\right) \\
& \gamma\left(q_{2}\right) A k_{1}\left(q_{\imath}\right)+\delta\left(q_{\imath}\right) A k_{2}\left(q_{2}\right)=k_{2}\left(p_{\imath}\right),
\end{aligned}
$$

$$
\left(\begin{array}{ll}
\alpha & \beta \\
\gamma & \delta
\end{array}\right)=\left(\begin{array}{ll}
a & b \\
c & d
\end{array}\right)^{-1}
$$

and $a, b, c, d: N_{0} \rightarrow \boldsymbol{R}$ are given by

$$
\begin{aligned}
& A k_{1}\left(q_{\imath}\right)={ }^{t}\left(*, \cdots, *, a\left(q_{\imath}\right), b\left(q_{\imath}\right)\right) \\
& A k_{2}\left(q_{\imath}\right)={ }^{t}\left(*, \cdots, *, c\left(q_{\imath}\right), d\left(q_{\imath}\right)\right) .
\end{aligned}
$$

Let $V^{n+3}=\boldsymbol{R}^{n-1} \times \boldsymbol{R}_{2}^{4}$, where $\boldsymbol{R}_{2}^{4}=\operatorname{span}\left({ }^{t}(\mathbf{0}, 1,0,0,0),{ }^{t}(\mathbf{0}, 0,1,0,0),{ }^{t}(\mathbf{0}, 0,0,1,0)\right.$, $\left.{ }^{\boldsymbol{t}}(\mathbf{0}, 0,0,0,1)\right)$. Then since $\operatorname{span}_{1 \leqq \imath 8}\left(k_{1}\left(q_{\imath}\right), k_{2}\left(q_{\imath}\right)\right)=\boldsymbol{R}_{2}^{4}$ and $\underset{1 \leqq \imath \geqq 8}{\operatorname{span}}\left(A k_{1}\left(q_{2}\right), A k_{2}\left(q_{\imath}\right)\right)=$ $\boldsymbol{R}_{2}^{4}$, we get

$$
A=\left(\begin{array}{ll}
A_{1} & 0 \\
B & A_{2}
\end{array}\right)
$$

From Lemma 3.1, it follows $B=0$ and $A_{1} \in O(n-1)$. Now, if we check (9.1) 
carefully, we get $A_{2}= \pm$ idendity.

q.e.d.

Now, our image hypersurface of $M$ by $A_{p}$ has the regular octagon at the image point of $p$ as was shown in $\S 8$. Thus for $M^{\prime}$ to be a Lie image of an isoparametric hypersurface, this image by $A_{p}$ itself must be isoparametric. Unfortunately, we can not show this without additional assumption on $M$.

THEOREM II. Let $M$ be a compact embedded Dupin hypersurface with four principal curvatures $\lambda>\mu>\nu>\tau$ in $\bar{M}(c)$. If $[\lambda, \tau ; \mu, \nu]$ is constant on $M$, and if for each $\lambda$-leaf $L^{\lambda}$ ( $\tau$-leaf $L^{\tau}$, resp.), there exists a $\nu$-leaf $L_{\lambda}^{\nu}\left(\mu\right.$-leaf $L_{\tau}^{\mu}$, resp.) such that $L_{q}^{\mu} \cap L_{\lambda}^{\nu} \neq \varnothing$ for all $q \in L^{\lambda}\left(L_{q}^{\nu} \cap L_{\tau}^{\mu} \neq \varnothing\right.$ for all $q \in L^{\tau}$, resp.), then $M^{\prime}$ is Lie equivalent to an isoparametric hypersurface in a sphere.

Now, we prove this theorem. For simplicity, we denote by $\tilde{M}$ the image hypersurface of $M$ by $A_{p}$, on which the relation in Fig. 9.1 is satisfied.

LEMMA 9.2. For $L_{1}^{\lambda}, L_{\lambda}^{\nu}=L_{4}^{\nu}$. Similarly, for $L_{3}^{\lambda}\left(L_{5}^{\lambda}, L_{7}^{\lambda}\right.$, resp. $), L_{\lambda}^{\nu}=L_{1}^{\nu}\left(L_{3}^{\nu}\right.$, $L_{2}^{\nu}$, resp.), and for $L_{2}^{\tau}\left(L_{4}^{\tau}, L_{6}^{\tau}, L_{8}^{\tau}\right.$, resp.), $L_{\tau}^{\mu}=L_{5}^{\mu}\left(L_{2}^{\mu}, L_{1}^{\mu}, L_{3}^{\mu}\right.$, resp. $)$.

Proof. Suppose that $L_{\lambda}^{\nu} \neq L_{4}^{\nu}$. Let $q_{1} \in L_{1}^{\mu} \cap L_{\lambda}^{\nu}$ and $q_{2} \in L_{2}^{\lambda} \cap L_{\lambda}^{\nu}$. Then we can write

$$
\begin{aligned}
& q_{1}=c_{1}^{\mu}+\left\|p_{1}-c_{1}^{\mu}\right\| \sin \varphi u+\cos \varphi\left(p_{1}-c_{1}^{\mu}\right), \\
& q_{2}=c_{2}^{\mu}+\left\|p_{2}-c_{2}^{\mu}\right\| \sin \varphi v+\cos \varphi\left(p_{2}-c_{2}^{\mu}\right),
\end{aligned}
$$

for some $u \in T_{p_{1}} L_{1}^{\mu}$ and $v \in T_{p_{2}} L_{2}^{\mu}$, where, denoting by $n_{\imath}$ the unit normal vector to $\tilde{M}$ at $p_{\imath}$, we know (see Remark 6.2, and (2.2) of [5]),

$$
c_{i}^{\mu}=p_{i}+\frac{H_{i}^{\mu}}{\left\|H_{i}^{\mu}\right\|^{2}}=p_{i}+\frac{\mu\left(p_{i}\right) n_{i}-p_{i}}{\mu\left(p_{i}\right)^{2}+1}, \quad i=1,2 .
$$

Obviously $\varphi \neq 0 \neq \phi$, and by $L_{\lambda}^{\nu} \neq L_{4}^{\nu}, \varphi \neq \pi \neq \phi$. Note that the focal point of $L_{i}^{\mu}$ is given by

$$
f_{i}^{\mu}=\cos \frac{3 \pi}{8} p_{i}+\sin \frac{3 \pi}{8} n_{\imath}, \quad i=1,2 .
$$

Now, let $f^{\nu}$ be the focal point of $L_{\lambda}^{\nu}$. Then since $q_{i}$ lies on $f_{i}^{\mu} f^{\nu}, q_{1}$ and $q_{2}$ lies on the geodesic two sphere determined by $f_{1}^{\mu}, f_{2}^{\mu}$ and $f^{\nu}$. Since we have

$$
\begin{aligned}
& f_{i}^{\mu} \in \operatorname{span}\left(p_{1}, n_{1}\right)=\operatorname{span}\left(p_{2}, n_{2}\right) \\
& q_{1} \in \operatorname{span}\left(p_{1}, n_{1}, u\right) \\
& q_{2} \in \operatorname{span}\left(p_{2}, n_{2}, v\right)=\operatorname{span}\left(p_{1}, n_{1}, v\right),
\end{aligned}
$$

we conclude that $u$ and $v$ should be parallel along $\gamma$. But then apparently, $L_{1}^{\mu}$ would intersect $L_{2}^{\mu}$, a contradiction. Thus we have $L_{\lambda}^{\nu}=L_{4}^{\nu}$. q.e.d. 
Proposition 9.3. Among tangent spaces of leaves at $p_{i}^{\prime} s, T L_{1}^{\lambda}, T L_{3}^{\nu}, T L_{4}^{\nu}$, $T L_{5}^{\lambda}$ are parallel with respect to the connection of $S^{n}$. Other parallel families are $\left\{T L_{2}^{\tau}, T L_{1}^{\mu}, T L_{8}^{\mu}, T L_{7}^{\tau}\right\},\left\{T L_{3}^{\lambda}, T L_{2}^{\nu}, T L_{1}^{\nu}, T L_{7}^{\lambda}\right\}$ and $\left\{T L_{4}^{\tau}, T L_{3}^{\mu}, T L_{2}^{\mu}, T L_{1}^{\tau}\right\}$.

Proof. Put $T L_{1}^{\lambda}=X, T L_{1}^{\mu}=Y, T L_{1}^{\nu}=Z, T L_{1}^{\tau}=W$. In the following, "=" means "be parallel to" with respect to the connection of $S^{n}$. Note that $L_{1}^{\lambda} \ni q \rightarrow$ $L_{q}^{\mu} \cap L_{4}^{\nu} \in L_{4}^{\nu}$ is an onto diffeo because of $\operatorname{dim} L_{1}^{\lambda}=\operatorname{dim} L_{4}^{\nu}=m_{1}$. Since $f_{1}^{\lambda}$ and $f_{q}^{\mu}$ lies on the normal geodesic $\gamma_{q}$ at $q$, and since $f_{4}^{\nu}=-f_{1}^{\lambda}, L_{q}^{\mu} \cap L_{4}^{\nu}$ also lies on $\gamma_{q}$. Thus $L_{4}^{\nu}$ is contained in the $\left(m_{1}+1\right)$-dimensional geodesic sphere determined by $f_{1}^{\lambda}$ and $L_{1}^{\lambda}$, and obviously intersects $\gamma_{q}$ orthogonally for any $q \in L_{1}^{\lambda}$. Therefore we conclude that $T L_{4}^{\nu}=T L_{1}^{\lambda}$. Similarly, we get $T L_{6}^{\tau}=Y, T L_{3}^{\lambda}=Z, T L_{3}^{\mu}=W$. Then from $T_{\imath} M:=T_{p_{i}} M=T_{p_{1}} M$, it follows that $T L_{4}^{\tau}=W, T L_{6}^{\lambda}=X$ and so $T L_{2}^{\mu}$ $=W, T L_{3}^{\nu}=X$. Thus we get $T L_{2}^{\tau}=Y, T L_{2}^{\nu}=Z$ and so $T L_{5}^{\mu}=Y, T L_{7}^{\lambda}=Z$.

q.e.d.

Remark 9.4. Without the assumption on leaves, there are infinitely many cases in the relation among tangent spaces of leaves.

By Proposition 9.3, we see that for any point $q \in L_{1}^{\lambda}$, the normal geodesic $\gamma_{q}$ cuts $\tilde{M}$ orthogonally at eight points $q_{1}=q, q_{2}, \cdots, q_{8}$, numbered in order along $\gamma_{q}$, where $q_{1}, q_{2} \in L_{1}^{\lambda}, q_{3}, q_{8} \in L_{3}^{\nu}, q_{4}, q_{7} \in L_{4}^{\nu}$ and $q_{5}, q_{6} \in L_{6}^{\lambda}$. In the same way, for any point $q \in L_{\imath}^{*}, *=\lambda, \mu, \nu, \tau$, and $i=1,2, \cdots, 8$, it holds that, the intersection of the normal geodesic $\gamma_{q}$ with $\tilde{M}$ makes a regular octagon. Note that at such points of intersection, all $\Lambda_{\alpha \alpha}^{\beta}=0$, for any $\alpha, \beta$ such that $\alpha \notin[\beta]$ (see Remark 6.2). Thus we have:

Proof of Theorem II. Let $x$ be any point of $\tilde{M}$. Then we can find $x_{1} \in$ $L_{1}^{\lambda}, x_{2} \in L_{x_{1}}^{\mu}$ and $x_{3} \in L_{x_{2}}^{\nu}$ such that $x \in L_{x_{3}}^{\tau}$. At $x_{1}$, the relation of the normal geodesic and $\tilde{M}$ is the same as in Fig. 9.1 where we replace $p_{1}$ by $x_{1}$ and other points of intersection by suitable symboles. Next at $x_{2}$, and then at $x_{3}$, we can show the same thing. Finally at $x$, we conclude that $\Lambda_{\alpha \alpha}^{\beta}(x)=0$ for all $\alpha$ and $\beta$ such that $\alpha \notin[\beta]$. Thus the Lie image of the Dupin hypersurface $M$ by $A_{p}=A_{5} \cdot A_{4} \cdot A_{3} \cdot C \cdot A_{2} \cdot A_{1}$ is isoparametric.

q.e.d.

\section{REFERENCES}

[1] Cecil, T. And Chern, S.S., Tautness and Lie sphere geometry, Math. Ann. 278 (1987), 381-399.

[2] Cecil, T. AND Ryan, P., Focal sets, taut embeddings and the cyclides of Dupin, Math. Ann. 236 (1978), 177-190.

[3] Cecil, T. ANd Ryan, P., Tight and taut immersions of manifolds, Reseach Notes in Mathematics 107, Pitman, London (1985).

[4] Kaneko, J., Wave equation and Dupin hypersurface, Memoirs of the Fac. of Sci., Kyushu Univ. Ser. A, 40 (1985), 51-55.

[5] MryaOKA, R., Compact Dupin hypersurfaces with three principal curvatures, 
Math. Zeit. 187 (1984), 433-452.

[6] Mryaoka, R. and Ozawa, T., Construction of taut embeddings and Cecil-Ryan conjecture, to appear in Proc. of Symp. in Diff. Geom. 1988.

[7] MüNZNER, H.F., Isoparametrische Hyperflächen in Sphären I, Math. Ann. 251 (1980), 57-71.

[8] Pinkall, U., Dupin hypersurfaces, Preprint (1983).

[9] Pinkall, U., Dupin hypersurfaces, Math. Ann. 270 (1985), 427-440.

[10] Pinkall, U. and Thorbergsson, G., Deformations of Dupin Hypersurfaces, to appear in Proc. of A.M.S.

[11] Thorbergsson, G., Dupin hypersurfaces, Bull London Math. Soc. 15 (1983), 493-498.

Department of Mathematics

ToKyo Institute of Technology

OHokayama, Meguro-ku

152 TOKYO, JAPAN 